\title{
Conformal Structures Associated to Generic Rank 2 Distributions on 5-Manifolds - Characterization and Killing-Field Decomposition ${ }^{\star}$
}

Matthias HAMMERL and Katja SAGERSCHNIG

Faculty of Mathematics, University of Vienna, Nordbergstrasse 15, 1090 Vienna, Austria

E-mail: matthias.hammerl@univie.ac.at,katja.sagerschnig@univie.ac.at

Received April 09, 2009, in final form July 28, 2009; Published online August 04, 2009

doi:10.3842/SIGMA.2009.081

\begin{abstract}
Given a maximally non-integrable 2-distribution $\mathcal{D}$ on a 5-manifold $M$, it was discovered by P. Nurowski that one can naturally associate a conformal structure $[g]_{\mathcal{D}}$ of signature $(2,3)$ on $M$. We show that those conformal structures $[g]_{\mathcal{D}}$ which come about by this construction are characterized by the existence of a normal conformal Killing 2-form which is locally decomposable and satisfies a genericity condition. We further show that every conformal Killing field of $[g]_{\mathcal{D}}$ can be decomposed into a symmetry of $\mathcal{D}$ and an almost Einstein scale of $[g]_{\mathcal{D}}$.
\end{abstract}

Key words: generic distributions; conformal geometry; tractor calculus; Fefferman construction; conformal Killing fields; almost Einstein scales

2000 Mathematics Subject Classification: 34A26; 35N10; 53A30; 53B15; 53B30

Dedicated to Peter Michor on the occasion of his 60th birthday celebrated at the Central European Seminar in Mikulov, Czech Republic, May 2009.

\section{Introduction and statement of results}

In this section we briefly introduce the main objects of interest and state the results of this text.

\subsection{Generic rank 2-distributions on 5-manifolds}

Let $M$ be a smooth 5-dimensional manifold and consider a subbundle $\mathcal{D}$ of the tangent bundle $T M$ which shall be of constant rank 2 . We say that $\mathcal{D}$ is generic if it is maximally nonintegrable in the following sense: For two subbundles $\mathcal{D}_{1} \subset T M$ and $\mathcal{D}_{2} \subset T M$ we define

$$
\left[\mathcal{D}_{1}, \mathcal{D}_{2}\right]_{x}:=\operatorname{span}\left(\left\{[\xi, \eta]_{x}: \xi \in \Gamma\left(\mathcal{D}_{1}\right), \eta \in \Gamma\left(\mathcal{D}_{2}\right)\right\}\right) .
$$

Then we demand that $[\mathcal{D}, \mathcal{D}] \subset T M$ is a subbundle of constant rank 3 and $[\mathcal{D},[\mathcal{D}, \mathcal{D}]]=T M$. In other words, two steps of taking Lie brackets of sections of $\mathcal{D}$ yield all of $T M$.

It is a classical result of Élie Cartan [19] that generic rank 2-distributions on $M$ can equivalently be described as parabolic geometries of type $\left(G_{2}, P\right)$. This will be explained in Section 4 .

\subsection{The associated conformal structure of signature $(2,3)$ and its characterization}

In [36] P. Nurowski used Cartan's description of generic distributions to associate to every such distribution a conformal class $[g]_{\mathcal{D}}$ of signature $(2,3)$-metrics on $M$.

\footnotetext{
*This paper is a contribution to the Special Issue "Élie Cartan and Differential Geometry". The full collection is available at http://www.emis.de/journals/SIGMA/Cartan.html
} 
There is a well studied result similar to Nurowski's construction: This is the classical Fefferman construction $[22,4,6,12,13,34,35]$ of a (pseudo) conformal structure on an $S^{1}$-bundle over a $C R$ manifold. It has been observed by A. Cap in [8] that both Nurowski's and Fefferman's results admit interpretations as special cases of a more general construction relating parabolic geometries of different types. In Section 4.4 we will discuss conformal structures associated to rank two distributions in this picture. Furthermore, we prove that given a holonomy reduction of a conformal structure $[g]$ of signature $(2,3)$ to a subgroup of $G_{2}$, the conformal class $[g]$ is induced by a distribution $\mathcal{D} \subset T M$.

Using strong techniques from the BGG-machinery [16, 5, 29] and tractor bundles [11, 10], we then proceed to prove our first main result in Section 5. Before we can state it we introduce some simple notation for tensorial expressions; this is slightly redundant since we will later use a form of index notation for such formulas. Take some $g \in[g]$ and let $\eta \in \otimes^{k} T^{*} M$ for $k \geq 2$. The trace over the $i$-th and the $j$-th slot of $\eta$ via the (inverse of) the metric $g$ will be denoted $\operatorname{tr}_{i, j}(\eta) \in \otimes^{k-2} T^{*} M$. For an arbitrary tensor $\eta$, alt $(\eta)$ is the full alternation of $\eta$.

Theorem A. Let $[g]$ be a conformal class of signature $(2,3)$ metrics on $M$. Then $[g]$ is induced from a generic rank 2 distribution $\mathcal{D} \subset T M$ if and only if there exists a normal conformal Killing 2 -form $\phi$ that is locally decomposable and satisfies the following genericity condition: Let $g \in[g]$ be a metric in the conformal class, D its Levi-Civita connection and P its Schouten tensor (11). Define

$$
\begin{aligned}
\mu:= & \operatorname{tr}_{1,2} D \phi \in T^{*} M, \\
\rho:= & +2 \triangle \phi+4 \operatorname{alt}\left(\operatorname{tr}_{1,3} D D \phi\right)+3 \operatorname{alt}\left(\operatorname{tr}_{2,3} D D \phi\right) \\
& +24 \operatorname{alt}\left(\operatorname{tr}_{1,3} P \otimes \phi\right)-6 \operatorname{tr}_{1,2} P \otimes \phi \in \Lambda^{2} T^{*} M .
\end{aligned}
$$

Here we use the convention

$$
\triangle \sigma=-\operatorname{tr}_{1,2} D D \sigma .
$$

Then one must have

$$
\phi \wedge \mu \wedge \rho \neq 0 .
$$

To be precise, Theorem A assumes orientability of $T M$, but this is a minor assumption only made for convenience of presentation - see Remark 5 .

\subsection{Killing field decomposition}

Let $\operatorname{sym}(\mathcal{D})$ denote the infinitesimal symmetries of the distribution $\mathcal{D}$, i.e.,

$$
\operatorname{sym}(\mathcal{D})=\left\{\xi \in \mathfrak{X}(M): \mathcal{L}_{\xi} \eta=[\xi, \eta] \in \Gamma(\mathcal{D}) \forall \eta \in \Gamma(\mathcal{D})\right\} .
$$

The corresponding objects for conformal structures are the conformal Killing fields

$$
\mathbf{c K f}([g])=\left\{\xi \in \mathfrak{X}(M): \mathcal{L}_{\xi} g=e^{2 f} g \text { for some } g \in[g] \text { and } f \in \mathrm{C}^{\infty}(M)\right\} .
$$

Since the construction that associates a conformal structure $[g]_{\mathcal{D}}$ to a distribution $\mathcal{D}$ is natural, every symmetry $\xi \in \mathfrak{X}(M)$ of the distribution will also preserve the associated conformal structure $[g]_{\mathcal{D}}$, i.e., it is a conformal Killing field. This yields an embedding

$$
\operatorname{sym}(\mathcal{D}) \hookrightarrow \operatorname{cKf}\left([g]_{\mathcal{D}}\right) .
$$

We will show that a complement to $\operatorname{sym}(\mathcal{D})$ in $\mathbf{c K f}\left([g]_{\mathcal{D}}\right)$ is given by the almost Einstein scales of $[g]_{\mathcal{D}}$ : a function $\sigma \in \mathrm{C}^{\infty}(M)$ is an almost Einstein scale for $g \in[g]_{\mathcal{D}}$ if it is non-vanishing on an open dense subset $U$ of $M$ and satisfies that $\sigma^{-2} g$ is Einstein on $U$ [24]. The natural origin of almost Einstein scales via tractor calculus will be seen in Section 3. 
Theorem B. Let $[g]_{\mathcal{D}}$ be the conformal structure associated to a generic rank 2 distribution $\mathcal{D}$ on a 5-manifold $M$, and let $\phi$ be a conformal Killing form characterizing the conformal structure as in Theorem A. Then every conformal Killing field decomposes into a symmetry of the distribution $\mathcal{D}$ and an almost Einstein scale:

$$
\mathbf{c K f}\left([g]_{\mathcal{D}}\right)=\mathbf{s y m}(\mathcal{D}) \oplus \mathbf{a E s}\left([g]_{\mathcal{D}}\right) .
$$

The mapping that associates to a conformal Killing field $\xi \in \mathfrak{X}(M)$ its almost Einstein scale part with respect to the decomposition (2) is given by

$$
\xi \mapsto \operatorname{tr}_{1,3} \operatorname{tr}_{2,4}\left(\phi \otimes D \xi-\frac{1}{2} \xi \otimes D \phi\right),
$$

where $D$ is the Levi-Civita connection of an arbitrary metric $g$ in the conformal class.

The mapping that associates to an almost Einstein scale $\sigma \in \mathrm{C}^{\infty}(M)$ (for a metric $g \in[g]$ ) a conformal Killing field is given by

$$
\sigma \mapsto \operatorname{tr}_{2,3} \phi \otimes(D \sigma)-\frac{1}{4} \operatorname{tr}_{1,2}(D \phi) \sigma .
$$

We remark here that the constructions in this paper, both for characterization (Section 5) and automorphism-decomposition (Section 6), are largely analogous to the ones of [12] and [13] for the (classical) Fefferman spaces.

This article incorporates material of the authors' respective theses [39, 27].

\section{Preliminaries on Cartan and parabolic geometries}

In this section we discuss general parabolic geometries. These are special kinds of Cartan geometries.

\subsection{Cartan geometries}

Let $G$ be a Lie group and $P<G$ a closed subgroup. The Lie algebras of $P$ and $G$ will be denoted by $\mathfrak{p}$ and $\mathfrak{g}$. Let $\mathcal{G} \stackrel{\pi}{\rightarrow} M$ be a $P$-principal bundle over a manifold $M$. The right action of $P$ on $\mathcal{G}$ will be denoted by $r^{p}(u)=u \cdot p$ for $u \in \mathcal{G}$ and $p \in P$. The corresponding fundamental vector fields are $\zeta_{Y}(u):=\left.\frac{d}{d t}\right|_{t=0} r^{\exp (t Y)}(u)=\left.\frac{d}{d t}\right|_{t=0} u \cdot \exp (t Y)$ for $Y \in \mathfrak{p}$.

Definition 1. A Cartan geometry of type $(G, P)$ on a manifold $M$ is a $P$-principal bundle $\mathcal{G} \stackrel{\pi}{\rightarrow} M$ endowed with a Cartan connection form $\omega \in \Omega^{1}(\mathcal{G}, \mathfrak{g})$, i.e., a $\mathfrak{g}$-valued 1 -form on $\mathcal{G}$ satisfying

(C.1) $\omega_{u \cdot p}\left(T_{u} r^{p} \xi\right)=\operatorname{Ad}\left(p^{-1}\right) \omega_{u}(\xi)$ for all $p \in P, u \in \mathcal{G}$, and $\xi \in T_{u} \mathcal{G}$.

(C.2) $\omega\left(\zeta_{Y}\right)=Y$ for all $Y \in \mathfrak{p}$.

(C.3) $\omega_{u}: T_{u} \mathcal{G} \rightarrow \mathfrak{g}$ is an isomorphism for all $u \in \mathcal{G}$.

We say that $\omega$ is right-equivariant, reproduces fundamental vector fields and is an absolute parallelism $\omega: T \mathcal{G} \cong \mathcal{G} \times \mathfrak{g}$.

It is easily seen that for a Cartan geometry $(\mathcal{G}, \omega)$ of type $(G, P)$ the map $\mathcal{G} \times \mathfrak{g} \mapsto T M$ given by $(u, X) \mapsto T_{u} \pi \omega_{u}^{-1}(X)$ induces an isomorphism

$$
T M=\mathcal{G} \times_{P} \mathfrak{g} / \mathfrak{p} .
$$

In particular, $\operatorname{dim} M=\operatorname{dim} \mathfrak{g} / \mathfrak{p}$. 
Cartan geometries can be viewed as curved versions of homogeneous spaces: The homogeneous model of Cartan geometries of type $(G, P)$ is the principal bundle $G \rightarrow G / P$ endowed with the Maurer-Cartan form $\omega^{M C} \in \Omega^{1}(G, \mathfrak{g})$, which satisfies the Maurer-Cartan equation

$$
d \omega^{M C}(\xi, \eta)+\left[\omega^{M C}(\xi), \omega^{M C}(\eta)\right]=0
$$

for all $\xi, \eta \in \mathfrak{X}(G)$.

For a general Cartan geometry $(\mathcal{G}, \omega)$ the failure of $\omega$ to satisfy the Maurer-Cartan equation is measured by the curvature form $\Omega \in \Omega^{2}(\mathcal{G}, \mathfrak{g})$,

$$
\Omega(\xi, \eta)=d \omega(\xi, \eta)+[\omega(\xi), \omega(\eta)]
$$

One can show that $\Omega$ vanishes, i.e., $\omega$ is flat, if and only if $(\mathcal{G}, \omega)$ is locally isomorphic (in the obvious sense) with $\left(G, \omega^{M C}\right)$.

Since the Cartan connection defines an absolute parallelism $\omega: T \mathcal{G} \cong \mathcal{G} \times \mathfrak{g}$, its curvature can be equivalently encoded in the curvature function $\kappa \in \mathrm{C}^{\infty}\left(\mathcal{G}, \Lambda^{2}\left(\mathfrak{g}^{*}\right) \otimes \mathfrak{g}\right)$,

$$
\kappa(u)(X, Y):=\Omega\left(\omega_{u}^{-1}(X), \omega_{u}^{-1}(Y)\right) .
$$

One verifies that $\Omega$ vanishes on vertical fields $\zeta_{Y}$ for $Y \in \mathfrak{p}$, i.e., it is horizontal. This implies, that $\kappa$ in fact defines a function $\mathcal{G} \mapsto \Lambda^{2}(\mathfrak{g} / \mathfrak{p})^{*} \otimes \mathfrak{g}$. And since $\Omega$ is $P$-equivariant, so is $\kappa$.

We denote by $\mathcal{A} M:=\mathcal{G} \times{ }_{P} \mathfrak{g}$ the associated bundle corresponding to the restriction of the adjoint representation $\mathrm{Ad}: G \rightarrow \mathrm{GL}(\mathfrak{g})$ to $P$. It is called the adjoint tractor bundle (general tractor bundles will be introduced below).

Note that since the curvature of a Cartan connection is horizontal and $P$-equivariant, it factorizes to a $\mathcal{A} M$-valued 2-form $K \in \Omega^{2}(M, \mathcal{A} M)$ on $M$. Thus, $\Omega \in \Omega^{2}(\mathcal{G}, \mathfrak{g}), K \in \Omega^{2}(M, \mathcal{A} M)$ and $\kappa: \mathcal{G} \rightarrow \Lambda^{2}(\mathfrak{g} / \mathfrak{p})^{*} \otimes \mathfrak{g}$ all encode essentially the same object, namely the curvature of the Cartan connection form $\omega$, and technical reasons will determine which representation should be used at a given point.

\subsection{Tractor bundles}

For any $G$-representation $V$, the associated bundle

$$
\mathcal{V}:=\mathcal{G} \times{ }_{P} V
$$

is called a tractor bundle. Tractor bundles carry canonical linear connections: Extend the structure group of $\mathcal{G}$ from $P$ to $G$ by forming $\mathcal{G}^{\prime}:=\mathcal{G} \times{ }_{P} G$. Then $\omega$ extends uniquely to a $G$ equivariant $\mathfrak{g}$-valued 1-form $\omega^{\prime}$ on $\mathcal{G}$ reproducing fundamental vector fields - i.e., to a principal connection form. Since $\mathcal{V}=\mathcal{G} \times{ }_{P} V=\mathcal{G}^{\prime} \times_{G} V$ one has the induced tractor connection $\nabla^{\mathcal{V}}$ on $\mathcal{V}$. For computations we will use the following explicit formula: Let $\xi^{\prime} \in \mathfrak{X}(\mathcal{G})$ be a $P$-invariant lift of a vector field $\xi \in \mathfrak{X}(M)$ and $f_{s} \in \mathrm{C}^{\infty}(\mathcal{G}, V)^{P}$ be the $P$-equivariant $V$-valued function on $\mathcal{G}$ corresponding to $s \in \Gamma(\mathcal{V})$. Then $\nabla_{\xi}^{\mathcal{V}} s$ corresponds to the $P$-equivariant function

$$
\xi^{\prime} \cdot f_{s}+\omega\left(\xi^{\prime}\right) f_{s} .
$$

\subsection{Parabolic geometries}

We now specialize to parabolic geometries. This class of Cartan geometries has a natural algebraic normalization condition which is employed in this text to describe conformal structures and generic distributions as parabolic geometries. For a thorough discussion of parabolic geometries we refer to [15]. 
Let us start with the algebraic background and introduce the notion of a $|k|$-graded Lie algebra $\mathfrak{g}$ : this is a a semisimple Lie algebra together with a vector space decomposition $\mathfrak{g}=$ $\mathfrak{g}_{-k} \oplus \cdots \oplus \mathfrak{g}_{k}$ such that $\left[\mathfrak{g}_{i}, \mathfrak{g}_{j}\right] \subset \mathfrak{g}_{i+j}$. Then $\mathfrak{g}_{-}=\mathfrak{g}_{-k} \oplus \cdots \oplus \mathfrak{g}_{-1}$ and $\mathfrak{p}_{+}=\mathfrak{g}_{1} \oplus \cdots \oplus \mathfrak{g}_{k}$ are nilpotent subalgebras of $\mathfrak{g}$. The Lie algebra $\mathfrak{p}=\mathfrak{g}_{0} \oplus \cdots \oplus \mathfrak{g}_{k}$ is indeed a parabolic subalgebra of $\mathfrak{g}$, and $\mathfrak{g}_{0}$ is its reductive Levi part. The grading induces a filtration on $\mathfrak{g}$ via $\mathfrak{g}^{i}:=\mathfrak{g}_{i} \oplus \cdots \oplus \mathfrak{g}_{k}$.

Let $G$ be a Lie group with Lie algebra $\mathfrak{g}$. Let $P$ be a closed subgroup of $G$ whose Lie algebra is the parabolic $\mathfrak{p} \subset \mathfrak{g}$ and such that it preserves the filtration, i.e., for all $p \in P$ we have $\operatorname{Ad}(p) \mathfrak{g}^{i} \subset \mathfrak{g}^{i} \forall i \in \mathbb{Z}$.

Definition 2. A Cartan geometry of type $(G, P)$ for groups as introduced above is called a parabolic geometry.

In the following, we will also consider the subgroup

$$
G_{0}:=\left\{g \in P: \operatorname{Ad}(g) \mathfrak{g}_{i} \subset \mathfrak{g}_{i} \forall i \in \mathbb{Z}\right\}
$$

of all elements in $P$ preserving the grading on the Lie algebra, which has Lie algebra $\mathfrak{g}_{0}$, and the subgroup

$$
P_{+}:=\left\{p \in P:(\operatorname{Ad}(p)-\mathrm{id}) \mathfrak{g}^{i} \subset \mathfrak{g}^{i+1} \forall i \in \mathbb{Z}\right\},
$$

which has Lie algebra $\mathfrak{p}_{+}$. Actually $P$ decomposes as a semidirect product

$$
P=G_{0} \ltimes P_{+} ;
$$

thus $P / P_{+}=G_{0}$.

\subsection{Lie algebra differentials and normality}

Let $V$ be a $G$-representation. We now introduce algebraic differentials

$$
\partial: \Lambda^{i}(\mathfrak{g} / \mathfrak{p})^{*} \otimes V \rightarrow \Lambda^{i+1}(\mathfrak{g} / \mathfrak{p})^{*} \otimes V
$$

and

$$
\partial^{*}: \Lambda^{i+1}(\mathfrak{g} / \mathfrak{p})^{*} \otimes V \rightarrow \Lambda^{i}(\mathfrak{g} / \mathfrak{p})^{*} \otimes V .
$$

For the first of these, we use the $G_{0}$-equivariant identification of $\mathfrak{g}_{-}^{*}$ with $(\mathfrak{g} / \mathfrak{p})^{*}$ and define $\partial$ as the differential computing the Lie algebra cohomology of $\mathfrak{g}_{-}$with values in $V$. For the Kostant codifferential $\partial^{*}$ we use the $P$-equivariant identification of $(\mathfrak{g} / \mathfrak{p})^{*}$ with $\mathfrak{p}_{+}$given by the Killing form; it is then defined as the differential computing the Lie algebra homology of $\mathfrak{p}_{+}$with values in $V$. We include the explicit formula for $\partial^{*}$, which will be needed later on: On a decomposable element $\varphi=Z_{1} \wedge \cdots \wedge Z_{i} \otimes v \in \Lambda^{i} \mathfrak{p}_{+} \otimes V, \partial^{*}$ is given as

$$
\begin{aligned}
\partial^{*}(\varphi):= & \sum_{j=1}^{i}(-1)^{j} Z_{1} \wedge \cdots \wedge \widehat{Z_{j}} \wedge \cdots \wedge Z_{i} \otimes\left(X_{j} v\right) \\
& +\sum_{1 \leq j<k \leq i}(-1)^{j+k}\left[Z_{j}, Z_{k}\right] \wedge Z_{1} \wedge \cdots \wedge \widehat{Z_{j}} \wedge \cdots \wedge \widehat{Z_{k}} \wedge \cdots \wedge Z_{i} \otimes v .
\end{aligned}
$$

The $G$-representation $V$ carries a natural $G_{0}$-invariant grading $V_{0} \oplus \cdots \oplus V_{r}$ for some $r \in \mathbb{N}$. The induced filtration $V^{i}:=V_{i} \oplus \cdots \oplus V_{r}$ is even $P$-invariant. The grading on $V$ and the grading $\mathfrak{g}_{-k} \oplus \cdots \oplus \mathfrak{g}_{-1}=\mathfrak{g}_{-} \cong \mathfrak{g} / \mathfrak{p}$ naturally induce a grading on the spaces $C_{i}(V)=\Lambda^{i}(\mathfrak{g} / \mathfrak{p})^{*} \otimes V$, which is preserved by both $\partial$ and $\partial^{*}$. While $\partial^{*}$ is seen to be $P$-equivariant, $\partial$ is only $G_{0}$-equivariant. 
We consider the spaces of cocycles $Z_{i}(V):=\operatorname{ker} \partial^{*} \subset \Lambda^{i}(\mathfrak{g} / \mathfrak{p})^{*} \otimes V$, coboundaries $B_{i}(V):=$ $\operatorname{im} \partial^{*} \subset \Lambda^{i}(\mathfrak{g} / \mathfrak{p})^{*} \otimes V$ and cohomologies $H_{i}(V):=Z_{i}(V) / B_{i}(V)$. Let $\Pi_{i}: Z_{i}(V) \rightarrow H_{i}(V)$ be the canonical surjection. By [31], the differentials $\partial$ and $\partial^{*}$ are adjoint with respect to a natural inner product on the space $C_{i}(V)$. Via the Kostant Laplacian $\square=\partial^{*} \circ \partial+\partial \circ \partial^{*}$ this yields a $G_{0}$-invariant Hodge decomposition

$$
C_{i}(V)=\operatorname{im} \partial \oplus \operatorname{ker} \square \oplus \operatorname{im} \partial^{*} .
$$

Thus, as a $G_{0}$-module, $H_{i}(V)$ can be embedded into $Z_{i}(V) \subset C_{i}(V)$.

Since $\mathcal{G} \times{ }_{P} \mathfrak{g} / \mathfrak{p}=T M$ the associated bundle $\mathcal{C}_{i}:=\mathcal{G} \times{ }_{P} \Lambda^{i}(\mathfrak{g} / \mathfrak{p})^{*} \otimes V$ of $C_{i}(V)$ is $\Lambda^{i} T^{*} M \otimes \mathcal{V}$, whose sections are the $\mathcal{V}$-valued $i$-forms $\Omega^{i}(M, \mathcal{V})$. The $P$-equivariant differential $\partial^{*}$ carries over to the associated spaces,

$$
\partial^{*}: \Omega^{i+1}(M, \mathcal{V}) \rightarrow \Omega^{i}(M, \mathcal{V}) .
$$

We set $\mathcal{Z}_{i}(\mathcal{V}):=\mathcal{G} \times_{P} Z_{i}(V), \mathcal{B}_{i}(\mathcal{V}):=\mathcal{G} \times_{P} B_{i}(V)$ and $\mathcal{H}_{i}(\mathcal{V}):=\mathcal{G} \times_{P} H_{i}(V)$. The canonical surjection from $\mathcal{Z}_{i}(\mathcal{V})$ onto $\mathcal{H}_{i}(\mathcal{V})$ is denoted by $\Pi_{i}$. If the tractor bundle $\mathcal{V}$ in question is unambiguous we just write $\mathcal{C}_{i}, \mathcal{Z}_{i}, \mathcal{B}_{i}, \mathcal{H}_{i}$.

The Kostant codifferential provides a conceptual normalization condition for parabolic geometries: Recall that the curvature of a Cartan connection form $\omega$ factorizes to a two-form $K \in \Omega^{2}(M, \mathcal{A} M)$ on $M$ with values in the adjoint tractor bundle.

Definition 3. A Cartan connection form $\omega$ is called normal if $\partial^{*}(K)=0$. In this case one has the harmonic curvature $K_{H}=\Pi_{2}(K) \in \mathcal{H}_{2}(\mathcal{A} M)$.

In the picture of $P$-equivariant functions on $\mathcal{G}$, the harmonic curvature corresponds to the composition of the curvature function $\kappa$ with the projection $\Pi_{2}: Z_{2}(\mathfrak{g}) \rightarrow H_{2}(\mathfrak{g})$, i.e., to $\kappa_{H}=$ $\Pi_{2} \circ \kappa$.

There is a simple algorithm to compute the cohomology spaces $H_{i}(V)$ provided by Kostant's version of the Bott-Borel-Weil theorem, cf. [31, 41]. Mostly, we will just need to know $H_{0}(V)$, which turns out to be the lowest homogeneity of $V$, i.e., $H_{0}(V)=V / V^{1}=V /\left(\mathfrak{p}_{+} V\right)$.

\subsection{The BGG-(splitting-)operators}

The BGG-machinery developed in [16] and [5] will feature prominently at many crucial points in this paper. The presentation here is very brief and the most important operators will later be given explicitly (see the end of the next section on conformal geometry). The highly useful Lemma 1 below can be understood without its relation to the BGG-machinery.

The main observation is that for every $\sigma \in \Gamma\left(\mathcal{H}_{0}\right)$ there is a unique $s \in \Gamma(\mathcal{V})$ with $\Pi_{0}(s)=\sigma$ such that $\nabla^{\mathcal{V}} s \in \Gamma\left(\mathcal{Z}_{1}\right)$, i.e., such that $\partial^{*}\left(\nabla^{\mathcal{V}} s\right)=0$. This gives a natural splitting $L_{0}^{\mathcal{V}}$ : $\Gamma\left(\mathcal{H}_{0}\right) \rightarrow \Gamma(\mathcal{V})$ of $\Pi_{0}: \Gamma(\mathcal{V}) \rightarrow \Gamma\left(\mathcal{H}_{0}\right)$ called the 1-st BGG-splitting operator and it defines the 1-st $B G G$-operator

$$
\Theta_{0}^{\mathcal{V}}: \Gamma\left(\mathcal{H}_{0}\right) \rightarrow \Gamma\left(\mathcal{H}_{1}\right), \quad \sigma \mapsto \Pi_{1}\left(\nabla^{\mathcal{V}} s\left(L_{0}(\sigma)\right)\right) .
$$

We only remark that this construction of differential splitting operators of the projections $\Pi_{i}$ : $\mathcal{Z}_{i} \rightarrow \mathcal{H}_{i}$ proceeds similarly, and one obtains the celebrated BGG-sequence $\mathcal{H}_{i} \stackrel{\Theta_{i}}{\rightarrow} \mathcal{H}_{i+1}$.

We will often need the following consequence of the definition of $L_{0}^{\mathcal{V}}$ : If $s \in \Gamma(\mathcal{V})$ is parallel, one trivially has $\partial^{*}\left(\nabla^{\mathcal{V}} s\right)=0$, and thus $s=L_{0}^{\mathcal{V}}\left(\Pi_{0}(s)\right)$. This is important enough to merit a

Lemma 1. On the space of parallel sections of a tractor bundle $\mathcal{V}, L_{0}^{\mathcal{V}} \circ \Pi_{0}$ is the identity, i.e., if $s \in \Gamma(\mathcal{V})$ with $\nabla^{\mathcal{V}} s=0$, then

$$
s=L_{0}^{\mathcal{V}}\left(\Pi_{0}(s)\right) .
$$


In particular, if the projection of a parallel section $s \in \Gamma(\mathcal{V})$ to its part in $\Gamma\left(\mathcal{H}_{0}\right)=\Gamma\left(\mathcal{V} / \mathcal{V}^{1}\right)$ vanishes, s must already have been trivial.

We now proceed to discuss conformal structures as parabolic geometries in Section 3 and do likewise for generic rank two distributions in Section 4.

\section{Conformal structures}

Two pseudo-Riemannian metrics $g$ and $\hat{g}$ with signature $(p, q)$ on a $n=p+q$-dimensional manifold $M$ are said to be conformally equivalent if there is a function $f \in \mathrm{C}^{\infty}(M)$ such that $\hat{g}=e^{2 f} g$. The conformal equivalence class of $g$ is denoted by $[g]$ and $(M,[g])$ is said to be a manifold endowed with a conformal structure. An equivalent description of a conformal structure of signature $(p, q)$ is a a reduction of structure group of $T M$ to $\mathrm{CO}(p, q)=\mathbb{R}_{+} \times \mathrm{O}(p, q)$, and the corresponding $\mathrm{CO}(p, q)$-bundle will be denoted $\tilde{\mathcal{G}}_{0}$.

The associated bundle to $\tilde{\mathcal{G}}_{0}$ for the 1-dimensional representation $\mathbb{R}[w]$ of $\operatorname{CO}(p, q)$ given by

$$
(c, C) \in \mathrm{CO}(p, q)=\mathbb{R}_{+} \times O(p, q) \mapsto c^{w}
$$

for $w \in \mathbb{R}$ is called the bundle of conformal $w$-densities and denoted by $\mathcal{E}[w]$.

We will use abstract index notation and notation for weighted bundles similar to [25]: $\mathcal{E}_{a}:=$ $T^{*} M, \mathcal{E}^{a}:=T M, \mathcal{E}_{a b}=\mathcal{E}_{a} \otimes \mathcal{E}_{b}, \mathcal{E}_{a}[w]:=\mathcal{E}_{a} \otimes \mathcal{E}[w]$. Recall the Einstein convention, e.g., for $\xi^{a} \in \Gamma\left(\mathcal{E}^{a}\right)=\mathfrak{X}(M)$ and $\varphi_{a} \in \Gamma\left(\mathcal{E}_{a}\right)=\Omega^{1}(M), \xi^{a} \varphi_{a}=\varphi(\xi) \in \mathrm{C}^{\infty}(M)$. Round brackets will denote symmetrizations, e.g. $\mathcal{E}_{(a b)}=S^{2} T^{*} M$ and square brackets anti-symmetrizations, e.g. $\mathcal{E}_{[a b]}=\Lambda^{2} T^{*} M$. In the following we will not distinguish between the space of sections $\Gamma\left(\mathcal{E}_{a \cdots b}[w]\right)$ and $\mathcal{E}_{a \cdots b}[w]$ itself.

Given a metric $g \in[g]$, a section $\sigma \in \mathcal{E}[w]$ trivializes to a function $[\sigma]_{g} \in \mathrm{C}^{\infty}(M)$ and one has

$$
[\sigma]_{e^{2} f g}=e^{w f}[\sigma]_{g}
$$

Tautologically, the conformal class of metrics $[g]$ defines a canonical section $\boldsymbol{g}$ in $\mathcal{E}_{(a b)}[2]=$ $\Gamma\left(S^{2} T^{*} M \otimes \mathcal{E}[2]\right)$, called the conformal metric, such that the trivialization of $\boldsymbol{g}$ with respect to $g \in[g]$ is just $g$. The conformal metric $\boldsymbol{g}$ allows one to raise or lower indices with simultaneous adjustment of the conformal weight: e.g., for a vector field $\xi^{p} \in \mathcal{E}^{p}=\mathfrak{X}(M)$ one can form $\xi_{p}=\boldsymbol{g}_{p q} \xi^{q} \in \mathcal{E}_{p}[2]=\Gamma\left(T^{*} M \otimes \mathcal{E}[2]\right)$, which is a 1 -form of weight 2 .

\subsection{Conformal structures as parabolic geometries}

Let $M_{p, q}$ be a given symmetric bilinear form of signature $(p, q)$ on $\mathbb{R}^{n}=\mathbb{R}^{p, q}$, and define the symmetric bilinear form $h$ of signature $(p+1, q+1)$ on $\mathbb{R}^{n+2}$ by

$$
h=\left(\begin{array}{ccc}
0 & 0 & 1 \\
0 & M_{p, q} & 0 \\
1 & 0 & 0
\end{array}\right) .
$$

We define $\tilde{P} \subset \mathrm{SO}(h) \cong \mathrm{SO}(p+1, q+1)$ as the stabilizer of the isotropic ray $\mathbb{R}_{+} e_{1}$, and one finds $\tilde{P}=\mathrm{CO}(p, q) \ltimes \mathbb{R}^{n *}$. The Lie algebra $\mathfrak{s o}(p+1, q+1)=\mathfrak{s o}(h)$ is $|1|$-graded

$$
\mathfrak{s o}(h)=\mathfrak{s o}(h)_{-1} \oplus \mathfrak{s o}(h)_{0} \oplus \mathfrak{s o}(h)_{1}=\mathbb{R}^{n} \oplus \mathfrak{c o}(p, q) \oplus \mathbb{R}^{n *} .
$$

Realized in $\mathfrak{g l}(n+2)$ it is given by matrices of the form

$$
\left(\begin{array}{c|c|c}
-\alpha & -Z^{t} M_{p, q} & 0 \\
\hline X & A & Z \\
\hline 0 & -X^{t} M_{p, q} & \alpha
\end{array}\right), \quad \alpha \in \mathbb{R}, \quad X, Z \in \mathbb{R}^{n}, \quad A \in \mathfrak{s o}\left(M_{p, q}\right) .
$$


Let $(\tilde{\mathcal{G}} \rightarrow M, \tilde{\omega})$ be a Cartan geometry of type $(\mathrm{SO}(h), \tilde{P})$. Define

$$
\tilde{\mathcal{G}}_{0}:=\tilde{\mathcal{G}} / \tilde{P}_{+}=\tilde{\mathcal{G}} / \mathbb{R}^{n *} \text {. }
$$

Then $\tilde{\mathcal{G}}_{0}$ is a $\mathrm{CO}(p, q)$-principal bundle over $M$ and

$$
T M=\tilde{\mathcal{G}} \times_{\tilde{P}} \mathfrak{s o}(h) / \mathfrak{p}=\tilde{\mathcal{G}}_{0} \times_{\mathrm{CO}(p, q)} \mathbb{R}^{n},
$$

i.e., $\tilde{\mathcal{G}}_{0} \rightarrow M$ gives a reduction of structure group of $T M$ to $\mathrm{CO}(p, q)$ and thus a conformal structure of signature $(p, q)$.

Since there are many non-isomorphic Cartan geometries of type $(\mathrm{SO}(h), \tilde{P})$ describing the same conformal structure on the underlying manifold, one imposes a normalization condition on the curvature $K \in \Omega^{2}(M, \tilde{\mathcal{A}} M)$ of $\tilde{\omega}$. Using the notion of normality introduced in Definition 3, one has:

Theorem 1 ([18]). Up to isomorphism there is a unique $\tilde{P}$-principal bundle $\tilde{\mathcal{G}}$ over $M$ endowed with a normal Cartan connection form $\tilde{\omega} \in \Omega^{1}(\tilde{\mathcal{G}}, \mathfrak{s o}(h))$ such that $\tilde{\mathcal{G}} / \mathbb{R}^{n *}=\tilde{\mathcal{G}}_{0}$ is the conformal frame bundle of $(M,[g])$.

This provides an equivalence of categories between oriented conformal structures of signature $(p, q)$ and normal parabolic geometries of type $(\mathrm{SO}(h), \tilde{P})$.

\subsection{Tractor bundles for conformal structures}

The standard tractor bundle of conformal geometry is obtained by the associated bundle $\mathcal{T}:=$ $\tilde{\mathcal{G}} \times_{\tilde{P}} \mathbb{R}^{n+2}$ of the standard representation of $\tilde{P}=\mathrm{SO}(h)$ on $\mathbb{R}^{n+2}$. $\tilde{P}$ preserves the filtration

$$
\{0\} \subset\left(\begin{array}{c}
\mathbb{R} \\
0 \\
0
\end{array}\right) \subset\left(\begin{array}{c}
\mathbb{R} \\
\mathbb{R}^{n} \\
0
\end{array}\right) \subset\left(\begin{array}{c}
\mathbb{R} \\
\mathbb{R}^{n} \\
\mathbb{R}
\end{array}\right)
$$

of $\mathbb{R}^{n+2}$, and therefore gives a well-defined filtration $\{0\} \subset \mathcal{T}^{1} \subset \mathcal{T}^{0} \subset \mathcal{T}^{-1}=\mathcal{T}$. The associated graded of $\mathcal{T}$ is $\operatorname{gr}(\mathcal{T})=\operatorname{gr}_{-1}(\mathcal{T}) \oplus \operatorname{gr}_{0}(\mathcal{T}) \oplus \operatorname{gr}_{1}(\mathcal{T})$, with

$$
\begin{aligned}
& \operatorname{gr}_{1}(\mathcal{T}):=\mathcal{T}^{1}=\mathcal{E}[-1], \\
& \operatorname{gr}_{0}(\mathcal{T}):=\mathcal{T}^{0} / \mathcal{T}^{1}=\mathcal{E}_{a}[-1], \\
& \operatorname{gr}_{-1}(\mathcal{T}):=\mathcal{T}^{-1} / \mathcal{T}^{0}=\mathcal{E}[1] .
\end{aligned}
$$

It is a general and well known fact of conformal tractor calculus that a choice of metric $g \in[g]$ yields a reduction of the $\tilde{P}$-principal bundle $\tilde{\mathcal{G}}$ to the $\mathrm{CO}(p, q)$-principal bundle $\tilde{\mathcal{G}}_{0}$, and this is seen to provide an isomorphism of a natural bundle with its associated graded space. In the case of the standard tractor bundle $\mathcal{T}$, this gives an isomorphism of $\mathcal{T}$ with $\operatorname{gr}(\mathcal{T})$, and a section $s \in \Gamma(\mathcal{T})$ will then be written

$$
[s]_{g}=\left(\begin{array}{c}
\rho \\
\varphi_{a} \\
\sigma
\end{array}\right) \in\left(\begin{array}{c}
\mathcal{E}[-1] \\
\mathcal{E}_{a}[1] \\
\mathcal{E}[1]
\end{array}\right)
$$

For $\hat{g}=\mathrm{e}^{2 f} g$ one has the transformation

$$
[s]_{\hat{g}}=\left(\begin{array}{c}
\hat{\rho} \\
\hat{\varphi}_{a} \\
\hat{\sigma}
\end{array}\right)=\left(\begin{array}{c}
\rho-\Upsilon_{a} \varphi^{a}-\frac{1}{2} \sigma \Upsilon^{b} \Upsilon_{b} \\
\varphi_{a}+\sigma \Upsilon_{a} \\
\sigma
\end{array}\right)
$$


where $\Upsilon=d f$. The insertion of $\mathcal{E}[-1]$ into $\mathcal{T}$ as the top slot is independent of the choice of $g \in[g]$ and defines a section $\tau_{+} \in \mathcal{T}[1]$. The insertion of $\mathcal{E}[1]$ into $\mathcal{T}$ as the bottom slot is well defined only via a choice of $g \in[g]$ and defines a section $\tau_{-} \in \mathcal{T}[-1]$. Let $e_{1}, \ldots, e_{n+2}$ be the standard basis of $\mathbb{R}^{n+2}$. Then $\tau_{+}$and $\tau_{-}$can be understood as the sections corresponding to the constant functions on $\tilde{\mathcal{G}}_{0}$ mapping to $e_{1} \otimes 1 \in \mathbb{R}^{n+2} \otimes \mathbb{R}[1]$ resp. $e_{n+2} \otimes 1 \in \mathbb{R}^{n+2} \otimes \mathbb{R}[-1]$.

Since $h \in S^{2} T^{*} \mathbb{R}^{n+2}$ is $\mathrm{SO}(h)$-invariant it defines a tractor metric $\mathbf{h}$ on $\mathcal{T}$. With respect to $g \in[g]$ and the decomposition $(9)$ of an element $s \in \Gamma(\mathcal{T})$

$$
[\mathbf{h}]_{g}=\left(\begin{array}{ccc}
0 & 0 & 1 \\
0 & \boldsymbol{g} & 0 \\
1 & 0 & 0
\end{array}\right)
$$

Let $D$ be the Levi-Civita connection of $g \in[g]$, then the tractor connection $\nabla^{\mathcal{T}}$ on $\mathcal{T}$ is given by

$$
\left[\nabla_{c}^{\mathcal{T}} s\right]_{g}=\nabla_{c}^{\mathcal{T}}\left(\begin{array}{c}
\rho \\
\varphi_{a} \\
\sigma
\end{array}\right)=\left(\begin{array}{c}
D_{c} \rho-P_{c}^{b} \varphi_{b} \\
D_{c} \varphi_{a}+\sigma P_{c a}+\rho \boldsymbol{g}_{c a} \\
D_{c} \sigma-\varphi_{c}
\end{array}\right) .
$$

Here

$$
P=P(g)=\frac{1}{n-2}\left(\operatorname{Ric}(g)-\frac{\operatorname{Sc}(g)}{2(n-1)} g\right)
$$

is the Schouten tensor of $g$. The trace of the Schouten tensor is denoted $J=g^{p q} P_{p q}$.

The adjoint tractor bundle is $\tilde{\mathcal{A}} M=\tilde{\mathcal{G}} \times \tilde{P} \mathfrak{s o}(h)$, which can be identified with $\mathfrak{s o}(\mathcal{T}, \mathbf{h})=\Lambda^{2} \mathcal{T}$. With respect to $g \in[g], \tilde{\mathcal{A}} M=T M \oplus \mathfrak{c o}(T M, g) \oplus T^{*} M$, and in matrix notation a section $[s]_{g}=\xi \oplus(\alpha, A) \oplus \varphi \in \mathfrak{X}(M) \oplus \mathfrak{c o}(T M, g) \oplus \Omega^{1}(M)$ will be written as

$$
\left(\begin{array}{c|c|c}
-\alpha & -\varphi_{a} & 0 \\
\hline \xi^{a} & A & \varphi^{a} \\
\hline 0 & -\xi_{a} & \alpha
\end{array}\right)
$$

The curvature form $\tilde{K} \in \Omega^{2}(M, \tilde{\mathcal{A}} M)$ has in fact values in $\tilde{\mathcal{A}} M^{0}$; this is called torsion-freeness. It furthermore decomposes into Weyl curvature $C \in \Omega^{2}(M, \mathfrak{s o}(T M))=\mathcal{E}_{c_{1} c_{2}{ }^{c}{ }_{d}}$ and Cotton-York tensor $A \in \mathcal{E}_{a\left[c_{1} c_{2}\right]}$ :

$$
\tilde{K}_{c_{1} c_{2}}=\left(\begin{array}{ccc}
0 & -A_{a c_{1} c_{2}} & 0 \\
0 & C_{c_{1} c_{2} b}^{a} & A^{a}{ }_{c_{1} c_{2}} \\
0 & 0 & 0
\end{array}\right) .
$$

The Weyl curvature $C$ is the completely trace-free part of the Riemannian curvature $R$ of $g$. The Cotton-York tensor is given by $A=A_{a c_{1} c_{2}}=2 D_{\left[c_{1}\right.} P_{\left.c_{2}\right] a}$.

We will later need the first BGG-splitting operators for the tractor bundles $\mathcal{T}, \tilde{\mathcal{A}} M=\Lambda^{2} \mathcal{T}$ and $\Lambda^{3} \mathcal{T}$, and therefore give general formulas from [29] for the space $\mathcal{V}:=\Lambda^{k+1} \mathcal{T}$ for $k \geq 0$.

The $\tilde{P}$-invariant filtration $(7)$ of $\mathbb{R}^{n+2}$ from above carries over to the invariant filtration of the exterior power $V=\Lambda^{k+1} \mathbb{R}^{n+2}$, written $\{0\} \subset V^{1} \subset V^{0} \subset V^{-1}=V$. Again, this yields filtrations of the associated bundles: $\{0\} \subset \mathcal{V}^{1} \subset \mathcal{V}^{0} \subset \mathcal{V}^{-1}=\mathcal{V}:=\Lambda^{k+1} \mathcal{T}$. The notion of the associated graded space is the same: we define $\operatorname{gr}\left(\Lambda^{k} \mathcal{T}\right)$ as the direct sum over all $\operatorname{gr}_{i}\left(\Lambda^{k} \mathcal{T}\right):=\left(\Lambda^{k} \mathcal{T}\right)^{i} /\left(\Lambda^{k} \mathcal{T}\right)^{i+1}$. With respect to $g \in[g]$, for $k \geq 0$, one again obtains an isomorphism of $\Lambda^{k+1} \mathcal{T}$ with $\operatorname{gr}\left(\Lambda^{k+1} \mathcal{T}\right)$, and we will write

$$
\left[\Lambda^{k+1} \mathcal{T}\right]_{g}=\left(\begin{array}{c}
\mathcal{E}_{\left[a_{1} \cdots a_{k}\right]}[k-1] \\
\mathcal{E}_{\left[a_{1} \cdots a_{k+1}\right]}[k+1] \mid \mathcal{E}_{\left[a_{1} \cdots a_{k-1}\right]}[k-1] \\
\mathcal{E}_{\left[a_{1} \cdots a_{k}\right]}[k+1]
\end{array}\right)
$$


This identification employs the insertions of the top slot $\tau_{+} \in \mathcal{T}[1]$ and bottom slot $\tau_{-} \in \mathcal{T}[-1]$ :

$$
\left(\begin{array}{c}
\rho_{a_{1} \cdots a_{k}} \\
\varphi_{a_{0} \cdots a_{k}} \mid \mu_{a_{2} \cdots a_{k}} \\
\sigma_{a_{1} \cdots a_{k}}
\end{array}\right) \mapsto \tau_{-} \wedge \sigma+\varphi+\tau_{+} \wedge \tau_{-} \wedge \mu+\tau_{+} \wedge \rho
$$

To understand the map $\sigma \mapsto \tau_{-} \wedge \sigma$ better, observe via (8) that one has a canonical embedding of $\mathcal{E}_{\left[a_{1} \cdots a_{k}\right]}[k]=\Lambda^{k} \mathcal{E}_{a}[1]$ into $\left(\Lambda^{k} \mathcal{T}\right)^{0} /\left(\Lambda^{k} \mathcal{T}\right)^{1}=\operatorname{gr}_{0}\left(\Lambda^{k} \mathcal{T}\right)$. Since $\tau_{-} \in \mathcal{T}[-1], \sigma \mapsto \tau_{-} \wedge \sigma$ is thus seen to yield an isomorphism of $\mathcal{E}_{a_{1} \cdots a_{k}}[k+1]$ with $\mathrm{gr}_{-1}\left(\Lambda^{k+1} \mathcal{T}\right)$ and analogously for the other components.

The tractor connection on $\Lambda^{k+1} \mathcal{T}$ is given by

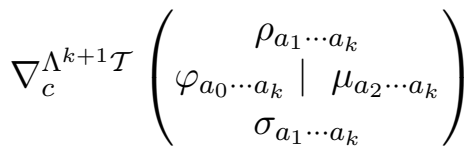

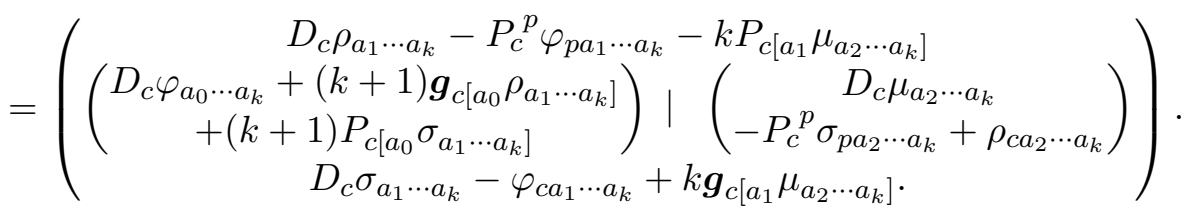

The first BGG-splitting operator $L_{0}^{\Lambda^{k+1} \mathcal{T}}: \mathcal{E}_{\left[a_{1} \cdots a_{k}\right]}[k+1] \rightarrow \Lambda^{k+1} \mathcal{T}$ is given by

$$
\begin{aligned}
& L_{0}^{\Lambda^{k+1} \mathcal{T}}(\sigma) \\
& \quad=\left(\begin{array}{c}
\left.-\frac{1}{n(k+1)} D^{p} D_{p} \sigma_{a_{1} \cdots a_{k}}+\frac{k}{n(k+1)} D^{p} D_{\left[a_{1}\right.} \sigma_{\left.|p| a_{2} \cdots a_{k}\right]}+\frac{k}{n(n-k+1)} D_{\left[a_{1}\right.} D^{p} \sigma_{\left.|p| a_{2} \cdots a_{k}\right]}\right) \\
+\frac{2 k}{n} P_{\left[a_{1}\right.}^{p} \sigma_{\left.|p| a_{2} \cdots a_{k}\right]}-\frac{1}{n} J \sigma_{a_{1} \cdots a_{k}} \\
D_{\left[a_{0}\right.} \sigma_{\left.a_{1} \cdots a_{k}\right]} \mid-\frac{1}{n-k+1} \boldsymbol{g}^{p q} D_{p} \sigma_{q a_{2} \cdots a_{k}} \\
\sigma_{a_{1} \cdots a_{k}}
\end{array}\right) .
\end{aligned}
$$

\subsection{Almost Einstein scales}

The first splitting operator for the standard tractor bundle is

$$
L_{0}^{\mathcal{T}}: \mathcal{E}[1] \rightarrow \Gamma(\mathcal{T}), \quad \sigma \mapsto\left(\begin{array}{c}
\frac{1}{n}(\triangle-J) \sigma \\
D \sigma \\
\sigma
\end{array}\right)
$$

By (10),

$$
\nabla^{\mathcal{T}} \circ L_{0}^{\mathcal{T}}(\sigma)=\left(\begin{array}{c}
\frac{1}{n} D_{c}(\triangle \sigma-J \sigma)-P_{c}^{p} D_{p} \sigma \\
\left(D_{a} D_{b} \sigma+P_{a b} \sigma\right)+\frac{1}{n}(\triangle \sigma-J \sigma) \boldsymbol{g}_{a b} \\
0
\end{array}\right) .
$$

Since $\frac{1}{n}(\triangle \sigma-J \sigma) \boldsymbol{g}_{a b}$ is minus the trace-part of $\left(D_{a} D_{b} \sigma+P_{a b} \sigma\right)$ and $\mathcal{H}_{1}(\mathcal{T})=\mathcal{E}_{(a b)_{0}}$ we have that the first BGG-operator of $\mathcal{T}$ is

$$
\Theta_{0}^{\mathcal{T}}: \mathcal{E}[1] \rightarrow \mathcal{E}_{(a b)_{0}}, \quad \sigma \mapsto\left(D_{a} D_{b} \sigma+P_{a b} \sigma\right)_{0} .
$$

By computing the change of the Schouten tensor $P$ with respect to a conformal rescaling one obtains that with $U=\{x \in M: \sigma(x) \neq 0\}$,

$$
\left(D_{a} D_{b} \sigma+P_{a b} \sigma\right)_{0}=0 \Leftrightarrow \sigma^{-2} \boldsymbol{g} \text { is Einstein on } U \text {. }
$$


This says that $P\left(\sigma^{-2} \boldsymbol{g}\right)$, or equivalently $\operatorname{Ric}\left(\sigma^{-2} \boldsymbol{g}\right)$, is a multiple of $\sigma^{-2} \boldsymbol{g}$ on $U$. $U$ always has to be an open dense subset of $M$, and we call the set of solutions of (17) the space of almost Einstein scales [24], i.e.

$$
\mathbf{a E s}([g])=\operatorname{ker} \Theta_{0}^{\mathcal{T}} \subset \mathcal{E}[1] .
$$

It turns out to be a differential consequence of (17) that $\frac{1}{n} D_{c}(\triangle \sigma-J \sigma)=P_{c}^{p} D_{p} \sigma$, and thus one has the well known fact

Proposition 1. $\nabla^{\mathcal{T}}$-parallel sections of the standard tractor bundle are in 1:1-correspondence with $\mathbf{a E s}([g])$.

\subsection{Conformal Killing forms}

Via (14) and (15) one computes that for $\sigma \in \mathcal{E}_{\left[a_{1} \cdots a_{k}\right]}[k+1]$ the projection of $\nabla^{\Lambda^{k+1}} \mathcal{T} \circ L_{0}^{\Lambda^{k+1}} \mathcal{T}(\sigma)$ to the lowest slot $\mathcal{E}_{c\left[a_{1} \cdots a_{k}\right]}[k+1]$ in $\Omega^{1}\left(M, \Lambda^{k+1} \mathcal{T}\right)$ is given by

$$
\sigma_{a_{1} \cdots a_{k}} \mapsto D_{c} \sigma_{a_{1} \cdots a_{k}}-D_{\left[a_{0}\right.} \sigma_{\left.a_{1} \cdots a_{k}\right]}-\frac{k}{n-k+1} \boldsymbol{g}^{p q} D_{p} \sigma_{q a_{2} \cdots a_{k}}
$$

This is the projection of $\sigma_{a_{1} \cdots a_{k}}$ to the highest weight part of $\mathcal{E}_{c\left[a_{1} \cdots a_{k}\right]}[k+1]$ which is formed by trace-free elements with trivial alternation, we write

$$
\mathcal{E}_{\left\{c\left[a_{1} \cdots a_{k}\right]\right\}_{0}}[k+1]:=\left\{\sigma_{a_{1} \cdots a_{k}}: 0=\sigma_{\left[c a_{1} \cdots a_{k}\right]} \text { and } 0=\boldsymbol{g}^{c a_{1}} \sigma_{c a_{1} \cdots a_{k}}\right\}
$$

One computes that in fact $\mathcal{H}_{1}^{\Lambda^{k+1} \mathcal{T}}=\mathcal{E}_{\left\{c\left[a_{1} \cdots a_{k}\right]\right\}_{0}}[k+1]$ and obtains the first BGG-operator

$$
\Theta_{0}^{\Lambda^{k+1} \mathcal{T}}: \mathcal{E}_{\left[a_{1} \cdots a_{k}\right]}[k+1] \rightarrow \mathcal{E}_{\left\{c\left[a_{1} \cdots a_{k}\right]\right\}_{0}}[k+1], \quad \sigma \mapsto D_{\{c} \sigma_{\left.a_{1} \cdots a_{k}\right\}_{0}} .
$$

Forms in the kernel of $\Theta_{0}^{\Lambda^{k+1} \mathcal{T}}$ are thus the conformal Killing k-forms.

Unlike the case of $k=0$, it is not true for $k \geq 1$ that always $\nabla^{\Lambda^{k+1}} \mathcal{T}\left(L_{0}^{\Lambda^{k+1}} \mathcal{T} \sigma\right)=0$ for $\sigma \in \operatorname{ker} \Theta_{0}^{\Lambda^{k+1} \mathcal{T}} \subset \mathcal{E}_{\left[a_{1} \ldots a_{k}\right]}[k+1]$. However, given a section $s \in \Gamma\left(\Lambda^{k+1} \mathcal{T}\right)$ with lowest slot $\Pi(s)=\sigma \in \mathcal{E}_{\left[a_{1} \cdots a_{k}\right]}$, one has by construction of $L_{0}^{\Lambda^{k+1} \mathcal{T}}$ that $s=L_{0}^{\Lambda^{k+1} \mathcal{T}} \sigma$ and that $\Theta_{0}^{\Lambda^{k+1} \mathcal{T}} \sigma=$ $\Pi \circ \nabla^{\Lambda^{k+1} \mathcal{T}} \circ L_{0}^{\Lambda^{k+1}} \mathcal{T}=0$; i.e., parallel sections of $\Lambda^{k+1} \mathcal{T}$ do always project to special solutions of $\Theta_{0}^{\Lambda^{k+1}} \sigma=0$. These solutions were termed normal conformal Killing forms by F. Leitner [33]. Thus, by definition, normal conformal Killing $k$-forms are in 1:1-correspondence (via $L_{0}^{\Lambda^{k+1} \mathcal{T}}$ and $\Pi$ ) with $\nabla^{\Lambda^{k+1}} \mathcal{T}$-parallel sections of $\Lambda^{k+1} \mathcal{T}$.

Denote the components of the splitting $L_{0}^{\Lambda^{k+1} \mathcal{T}} \sigma$ given in (15) by

$$
\left(\begin{array}{c}
\rho_{a_{1} \cdots a_{k}} \\
\varphi_{a_{0} \cdots a_{k}} \mid \\
\sigma_{a_{1} \cdots a_{k}}
\end{array}\right)
$$

A normal conformal Killing form satisfies $\nabla^{\Lambda^{k+1}} \mathcal{T}\left(L_{0}^{\Lambda^{k+1}} \mathcal{T} \sigma\right)$. By (14), the resulting equation in lowest slot just says that $\sigma$ is a conformal Killing form. Additionally, we get the following equations for the components $\varphi, \mu, \rho$ :

$$
\begin{aligned}
& D_{c} \rho_{a_{1} \cdots a_{k}}-P_{c}^{p} \varphi_{p a_{1} \cdots a_{k}}-k P_{c\left[a_{1}\right.} \mu_{\left.a_{2} \cdots a_{k}\right]}=0, \\
& D_{c} \varphi_{a_{0} \cdots a_{k}}+(k+1) \boldsymbol{g}_{c\left[a_{0}\right.} \rho_{\left.a_{1} \cdots a_{k}\right]}+(k+1) P_{c\left[a_{0}\right.} \sigma_{\left.a_{1} \cdots a_{k}\right]}=0, \\
& D_{c} \mu_{a_{2} \cdots a_{k}}-P_{c}^{p} \sigma_{p a_{2} \cdots a_{k}}+\rho_{c a_{2} \cdots a_{k}}=0 .
\end{aligned}
$$




\section{Generic rank two distributions and associated conformal structures}

\subsection{The distributions}

Let $M$ be a 5 -manifold. We are interested in generic rank 2 distributions on $M$, i.e., rank 2 subbundles $\mathcal{D} \subset T M$ such that values of sections of $\mathcal{D}$ and Lie brackets of two such sections span a rank 3 subbundle $[\mathcal{D}, \mathcal{D}]$ and values of Lie brackets of at most three sections span the entire tangent bundle $T M$ (recall (1)). In other words, these are distributions of maximal growth vector $(2,3,5)$ in each point.

Defining $T^{-1} M:=\mathcal{D}, T^{-2} M:=[\mathcal{D}, \mathcal{D}]$ and $T^{-3} M=T M$, the distribution gives rise to a filtration of the tangent bundle by subbundles compatible with the Lie bracket of vector fields in the sense that for $\xi \in \Gamma\left(T^{i} M\right)$ and $\eta \in \Gamma\left(T^{j} M\right)$ we have $[\xi, \eta] \in \Gamma\left(T^{i+j} M\right)$. Given such a filtration, the Lie bracket of vector fields induces a tensorial bracket $\mathcal{L}$ on the associated graded bundle $\operatorname{gr}(T M)=\bigoplus \operatorname{gr}_{i}(T M)$, where $\operatorname{gr}_{i}(T M)=T^{i} M / T^{i+1} M$. This bundle map $\mathcal{L}$ : $\operatorname{gr}(T M) \times \operatorname{gr}(T M) \rightarrow \operatorname{gr}(T M)$ is called the Levi bracket. It makes the bundle $\operatorname{gr}(T M)$ into a bundle of nilpotent Lie algebras; the fiber $\left(\operatorname{gr}(T M)_{x}, \mathcal{L}_{x}\right)$ is the symbol algebra at the point $x$. Note that a rank 2 distribution $\mathcal{D} \subset T M$ is generic if and only if the symbol algebra at each point is isomorphic to the graded Lie algebra $\mathfrak{g}=\mathfrak{g}_{-1} \oplus \mathfrak{g}_{-2} \oplus \mathfrak{g}_{-3}$, where $\operatorname{dim}\left(\mathfrak{g}_{-1}\right)=2, \operatorname{dim}\left(\mathfrak{g}_{-2}\right)=1$, $\operatorname{dim}\left(\mathfrak{g}_{-3}\right)=2$, and the only non-trivial components of the Lie bracket, $\mathfrak{g}_{-1} \times \mathfrak{g}_{-2} \rightarrow \mathfrak{g}_{-3}$ and $\Lambda^{2} \mathfrak{g}_{-1} \rightarrow \mathfrak{g}_{-2}$, define isomorphisms.

Generic rank 2 distributions in dimension 5 arise from ODEs of the form

$$
z^{\prime}=F\left(x, y, y^{\prime}, y^{\prime \prime}, z\right)
$$

with $\frac{\partial^{2} F}{\partial\left(y^{\prime \prime}\right)^{2}} \neq 0$ where $y$ and $z$ are functions of $x$, see e.g. [36] for that viewpoint. In his famous five-variables paper [19] from 1910, Élie Cartan associated to these distributions a canonical Cartan connection, a result that shall be stated more precisely in Section 4.4.

\subsection{Some algebra: $G_{2}$ in $\operatorname{SO}(3,4)$}

Let us recall one of the possible definitions of an exceptional Lie group of type $G_{2}$. It is well known (e.g. [3]) that the natural GL( $7, \mathbb{R}$ )-action on the space $\Lambda^{3} \mathbb{R}^{7^{*}}$ of 3 -forms on $\mathbb{R}^{7}$ has two open orbits and that the stabilizer of a 3 -form in either of these open orbits is a 14-dimensional Lie group. For one of these orbits it is a compact real form of the complex exceptional Lie group $G_{2}$, and for the other orbit it is a split real form. To distinguish between the two open orbits, consider the bilinear map $\mathbb{R}^{7} \times \mathbb{R}^{7} \rightarrow \Lambda^{7} \mathbb{R}^{7^{*}},(X, Y) \mapsto i_{X} \Phi \wedge i_{Y} \Phi \wedge \Phi$, associated to a 3 -form $\Phi$. This bilinear map is non-degenerate if and only if $\Phi$ is contained in an open orbit. In that case, it determines an invariant volume form vol on $\mathbb{R}^{7}$ given by the root ${ }^{9} \sqrt{D} \in \Lambda^{7}\left(\mathbb{R}^{7^{*}}\right)$ of its determinant $D \in\left(\Lambda^{7} \mathbb{R}^{7^{*}}\right)^{9}$, see e.g. [30]. Hence

$$
H(\Phi)(X, Y) \operatorname{vol}:=i_{X} \Phi \wedge i_{Y} \Phi \wedge \Phi
$$

defines a $\mathbb{R}$-valued bilinear form $H(\Phi)$ on $\mathbb{R}^{7}$ which is invariant under the action of the stabilizer of $\Phi$. It turns out that $H(\Phi)$ is positive definite if the stabilizer is the compact real form, and it has signature $(3,4)$ if the stabilizer is the split real form of $G_{2}$. In the sequel, let $G=G_{2}$ be the stabilizer of a 3 -form $\Phi \in \Lambda^{3} \mathbb{R}^{7^{*}}$ such that the associated bilinear form $H(\Phi)$ has signature $(3,4)$. The above discussion implies that this $G_{2}$ naturally includes into the special orthogonal group $\tilde{G}=\mathrm{SO}(3,4)$, an observation which will be crucial for what follows. 
Let us be more explicit and realize $\mathrm{SO}(3,4)$ as $\mathrm{SO}(h)$, with

$$
h=\left(\begin{array}{ccc}
0 & 0 & 1 \\
0 & M_{2,3} & 0 \\
1 & 0 & 0
\end{array}\right), \quad M_{2,3}=\left(\begin{array}{ccccc}
0 & 0 & 0 & 1 & 0 \\
0 & 0 & 0 & 0 & 1 \\
0 & 0 & -1 & 0 & 0 \\
1 & 0 & 0 & 0 & 0 \\
0 & 1 & 0 & 0 & 0
\end{array}\right)
$$

Via this bilinear form we identify $\mathbb{R}^{7^{*}} \cong \mathbb{R}^{7}$. Consider the standard basis $e_{1}, \ldots, e_{7}$ on $\mathbb{R}^{7}$, and define $G_{2}$ as the stabilizer of $\Phi \in \Lambda^{3} \mathbb{R}^{7}$,

$$
\begin{aligned}
\Phi:= & -\frac{1}{\sqrt{3}} e_{7} \wedge e_{2} \wedge e_{3}+\frac{1}{\sqrt{6}} e_{5} \wedge e_{4} \wedge e_{2}+\frac{1}{\sqrt{6}} e_{6} \wedge e_{4} \wedge e_{3} \\
& -\frac{1}{\sqrt{6}} e_{7} \wedge e_{4} \wedge e_{1}-\frac{1}{\sqrt{3}} e_{1} \wedge e_{5} \wedge e_{6} .
\end{aligned}
$$

Then, via the identification $\Lambda^{3} \mathbb{R}^{7^{*}} \cong \Lambda^{3} \mathbb{R}^{7}$,

$$
H(\Phi)(X, Y)=\frac{1}{\sqrt{6}} h(X, Y)
$$

and this equation characterizes the $\mathrm{SO}(h)$-conjugacy class of $G_{2}$. That is, $\Phi$ has $\mathrm{SO}(h)$-stabilizer conjugated to $G_{2}$ if and only if $H(\Phi)$ is some non-zero multiple of $h$.

The Lie algebra $\mathfrak{s o}(h)$ has the matrix representation (6). It contains the Lie algebra $\mathfrak{g}$ of $G_{2}$, which is formed by elements $M \in \mathfrak{g l}(7, \mathbb{R})$ such that $\Phi\left(M v, v^{\prime}, v^{\prime \prime}\right)+\Phi\left(v, M v^{\prime}, v^{\prime \prime}\right)+$ $\Phi\left(v, v^{\prime}, M v^{\prime \prime}\right)=0$, as the subalgebra consisting of matrices

$\left(\begin{array}{c|c|c|c|c}\operatorname{tr}(A) & Z & s & W & 0 \\ \hline X & A & \sqrt{2} \mathbb{J} Z^{t} & \frac{s}{\sqrt{2}} \mathbb{} & -W^{t} \\ \hline r & -\sqrt{2} X^{t} \mathbb{J} & 0 & -\sqrt{2} Z \mathbb{J} & s \\ \hline Y & -\frac{r}{\sqrt{2}} \mathbb{J} & \sqrt{2} \mathbb{J} X & -A^{t} & -Z^{t} \\ \hline 0 & -Y^{t} & r & -X^{t} & -\operatorname{tr}(A)\end{array}\right)$

with $A \in \mathfrak{g l}(2, \mathbb{R}), X, Y \in \mathbb{R}^{2}, Z, W \in \mathbb{R}^{2^{*}}, r, s \in \mathbb{R}$ and $\mathbb{J}=\left(\begin{array}{cc}0 & -1 \\ 1 & 0\end{array}\right)$.

For later use, let us note here that the complement of $\mathfrak{g}$ in $\mathfrak{s o}(h)$ with respect to the Killing form is isomorphic to the seven dimensional standard representation of $G_{2}$. That means we have a $G_{2}$-module decomposition

$$
\mathfrak{s o}(h)=\mathfrak{g} \oplus \mathbb{R}^{7} .
$$

The sequence

$$
0 \rightarrow \mathfrak{g} \hookrightarrow \mathfrak{s o}(h) \stackrel{i \Phi}{\rightarrow} \mathbb{R}^{7} \rightarrow 0
$$

is $G_{2}$-equivariant and exact. Here

$$
i \Phi: \mathfrak{s o}(h)=\Lambda^{2} \mathbb{R}^{7} \rightarrow \mathbb{R}^{7}
$$

is the insertion of $\mathfrak{s o}(h)$ into $\Phi$. The factor of $\Phi$ as given in (23) was chosen such that the insertion

$$
i \Phi: \mathbb{R}^{7} \rightarrow \Lambda^{2} \mathbb{R}^{7}=\mathfrak{s o}(h)
$$

splits sequence $(26)$.

Next we consider parabolic subgroups in $G_{2}$ and $\mathrm{SO}(h)$. Let $e_{1} \in \mathbb{R}^{7}$ be the first basis vector in the standard representation. Then the isotropy group of the ray $\mathbb{R}_{+} e_{1}$ is a parabolic 
subgroup $\tilde{P}$ in $\mathrm{SO}(h)$, and the intersection $P=\tilde{P} \cap G_{2}$ is a parabolic subgroup in $G_{2}$. To describe explicitly the corresponding parabolic subalgebra $\mathfrak{p} \subset \mathfrak{g}$, we introduce vector space decompositions of the Lie algebra. We consider the block decomposition

$$
\left(\begin{array}{ccccc}
\mathfrak{g}_{0} & \mathfrak{g}_{1} & \mathfrak{g}_{2} & \mathfrak{g}_{3} & 0 \\
\mathfrak{g}_{-1} & \mathfrak{g}_{0} & \mathfrak{g}_{1} & \mathfrak{g}_{2} & \mathfrak{g}_{3} \\
\mathfrak{g}_{-2} & \mathfrak{g}_{-1} & 0 & \mathfrak{g}_{1} & \mathfrak{g}_{2} \\
\mathfrak{g}_{-3} & \mathfrak{g}_{-2} & \mathfrak{g}_{-1} & \mathfrak{g}_{0} & \mathfrak{g}_{1} \\
0 & \mathfrak{g}_{-3} & \mathfrak{g}_{-2} & \mathfrak{g}_{-1} & \mathfrak{g}_{0}
\end{array}\right),
$$

of matrices (25); this defines a grading

$$
\mathfrak{g}=\mathfrak{g}_{-3} \oplus \mathfrak{g}_{-2} \oplus \mathfrak{g}_{-1} \oplus \mathfrak{g}_{0} \oplus \mathfrak{g}_{1} \oplus \mathfrak{g}_{2} \oplus \mathfrak{g}_{3}
$$

Note that the subalgebra $\mathfrak{g}_{-}=\mathfrak{g}_{-3} \oplus \mathfrak{g}_{-2} \oplus \mathfrak{g}_{-1}$ coincides with the symbol algebra of a generic rank two distribution in dimension five as explained in Section 4.1. The grading induces a filtration $\mathfrak{g}^{3} \subset \mathfrak{g}^{2} \subset \mathfrak{g}^{1} \subset \mathfrak{g}^{0} \subset \mathfrak{g}^{-1} \subset \mathfrak{g}^{-2} \subset \mathfrak{g}^{-3}$, which is preserved by the action of $P$ on $\mathfrak{g}$. The subalgebra $\mathfrak{p}=\mathfrak{g}^{0}$ is the Lie algebra of the parabolic $P$, and the subalgebra $\mathfrak{g}_{0} \cong \mathfrak{g l}(2, \mathbb{R})$ is the Lie algebra of the subgroup $G_{0} \subset P$ that even preserves the grading. The subgroup $G_{0}$ is isomorphic to $\mathrm{GL}_{+}(2, \mathbb{R})=\{M \in \mathrm{GL}(2, \mathbb{R}): \operatorname{det}(M)>0\}$.

\subsection{The homogeneous model and associated Cartan geometries}

Let us look at the Lie group quotient $G_{2} / P$ next. The action of $G_{2}$ on the class e $\tilde{P} \in \operatorname{SO}(h) / \tilde{P}$ induces a smooth map

$$
G_{2} / P \rightarrow \mathrm{SO}(h) / \tilde{P}
$$

Since both homogeneous spaces have the same dimension, the map is an open embedding. Since $G_{2} / P$ is a quotient of a semisimple Lie group by a parabolic subgroup, it is compact, and the map is in fact a diffeomorphism. The group $\mathrm{SO}(h)$ acts transitively on the space of null-rays in $\mathbb{R}^{7}$, which can be identified with the pseudo-sphere $Q_{2,3} \cong S^{2} \times S^{3}$. It turns out that the metric $h$ on $\mathbb{R}^{7}$ defined in (5) induces the conformal class of $\left(g_{2},-g_{3}\right)$ on $Q_{2,3}$, with $g_{2}, g_{3}$ being the round metrics on $S^{2}$ respectively $S^{3}$. The pullback of that conformal structure yields a $G_{2}$-invariant conformal structure on $G_{2} / P$.

Explicit descriptions of the canonical rank two distribution on $Q_{2,3} \cong S^{2} \times S^{3}$ can be found in [38]. In an algebraic picture the distribution corresponds to the $P$-invariant subspace $\mathfrak{g}^{-1} / \mathfrak{p} \subset \mathfrak{g} / \mathfrak{p}$. Via the identification of $T(G / P)$ with $G \times_{P} \mathfrak{g} / \mathfrak{p}$, this invariant subspace gives rise to a rank two distribution, which is generic in the sense of Section 4.1.

More generally, suppose $(\mathcal{G}, \omega)$ is any parabolic geometry of type $\left(G_{2}, P\right)$. Recall from Section 2 that the Cartan connection $\omega$ defines an isomorphism $T M \cong \mathcal{G} \times_{P} \mathfrak{g} / \mathfrak{p}$. Hence, for any such geometry, the subspace $\mathfrak{g}^{-1} / \mathfrak{p}$ gives rise to a rank two distribution. This distribution will be generic if a regularity condition on the Cartan connection is assumed; we shall introduce this condition next: Let

$$
T^{-1} M \subset T^{-2} M \subset T M
$$

be the sequence of subbundles of constant ranks 2,3 and 5 coming from the $P$-invariant filtration $\mathfrak{g}^{-1} / \mathfrak{p} \subset \mathfrak{g}^{-2} / \mathfrak{p} \subset \mathfrak{g} / \mathfrak{p}$. Consider the associated graded bundle $\operatorname{gr}(T M)$. This bundle can be naturally identified with $\mathcal{G} \times{ }_{P} \operatorname{gr}(\mathfrak{g} / \mathfrak{p}) \cong\left(\mathcal{G} / P_{+}\right) \times_{G_{0}} \mathfrak{g}_{-}$. Since the Lie bracket on the nilpotent Lie algebra $\mathfrak{g}_{-}$is invariant under the $G_{0}$-representation, it induces a bundle map $\{\}:, \operatorname{gr}(T M) \times$ $\operatorname{gr}(T M) \rightarrow \operatorname{gr}(T M)$, the algebraic bracket. A Cartan connection form $\omega$ is said to be regular if 
the filtration $T^{-1} M \subset T^{-2} M \subset T M$ is compatible with the Lie bracket of vector fields and the algebraic bracket coincides with the Levi bracket of the filtration. But this precisely means that the rank two subbundle $\mathcal{D}:=T^{-1} M$ is generic and $T^{-2} M=[\mathcal{D}, \mathcal{D}]$ (compare with Section 4.1 and the structure of $\mathfrak{g}_{-}$). Regularity can be expressed as a condition on the curvature of a Cartan connection. Since $\mathfrak{g}$ has a $P$-invariant filtration, we have a notion of maps in $\Lambda^{k}(\mathfrak{g} / \mathfrak{p})^{*} \otimes \mathfrak{g}$ of homogeneous degree $\geq l$, and the set of these maps is $P$-invariant. A Cartan connection form is regular if and only if the curvature function is homogeneous of degree $\geq 1$; this means that $\kappa(u)\left(\mathfrak{g}^{i}, \mathfrak{g}^{j}\right) \subset \mathfrak{g}^{i+j+1}$ for all $i, j$ and $u \in \mathcal{G}$. Note that if the curvature function takes values in $\Lambda^{2}(\mathfrak{g} / \mathfrak{p})^{*} \otimes \mathfrak{p}$, i.e. the geometry is torsion-free, then it is regular.

Now we can state Cartan's classical result in modern language. In this paper we restrict our considerations to orientable distributions. Equivalently, this means that the bundle $T M$ be orientable. Then we have the following:

Theorem 2 ([19]). One can naturally associate a regular, normal parabolic geometry $(\mathcal{G}, \omega)$ of type $\left(G_{2}, P\right)$ to an orientable generic rank two distribution in dimension five, and this establishes an equivalence of categories.

The above discussion explains that a regular parabolic geometry of type $\left(G_{2}, P\right)$ determines an underlying generic rank two distribution $\mathcal{D}$, and (for our choice of $P$ ) the distribution turns out to be orientable. The converse is shown in two steps: First one constructs a regular parabolic geometry inducing the given distribution. Next one employs an inductive normalization procedure based on the proposition below, which we state explicitly here, since it will be needed it in Proposition 4.

Proposition 2 ([15]). Let $(\mathcal{G}, \omega)$ be a regular parabolic geometry with curvature function $\kappa$, and suppose that $\partial^{*} \kappa$ is of homogeneous degree $\geq l$ for some $l \geq 1$. Then, there is a normal Cartan connection $\omega_{N} \in \Omega^{1}(\mathcal{G}, \mathfrak{g})$ such that $\left(\omega_{N}-\omega\right)$ is of homogeneous degree $\geq l$.

In the proposition the difference $\omega_{N}-\omega$, which is horizontal, is viewed as a function $\mathcal{G} \rightarrow$ $(\mathfrak{g} / \mathfrak{p})^{*} \otimes \mathfrak{g}$, and the homogeneity condition employs the canonical filtration of $(\mathfrak{g} / \mathfrak{p})^{*} \otimes \mathfrak{g}$.

\subsection{A Fefferman-type construction}

The relation in Section 4.3 between the homogeneous models $G_{2} / P$ and $\mathrm{SO}(h) / \tilde{P}$ suggests a relation between Cartan geometries of type $\left(G_{2}, P\right)$ and $(\mathrm{SO}(h), \tilde{P})$, i.e., generic rank two distributions and conformal structures. Indeed, it was P. Nurowski who first observed in [36] that any generic rank two distribution on a five manifold $M$ naturally determines a conformal class of metrics of signature $(2,3)$ on $M$. Starting from a system (21) of ODEs, he explicitly constructed a metric from the conformal class. A different construction of such a metric can be found in [14].

In the present paper, we shall discuss Nurowski's result as a special case of an extension functor of Cartan geometries, see [17, 8, 20]. Let $i^{\prime}: \mathfrak{g} \hookrightarrow \mathfrak{s o}(h)$ denote the derivative of the inclusion $i: G_{2} \hookrightarrow \mathrm{SO}(h)$. Given a Cartan geometry $(\mathcal{G} \rightarrow M, \omega)$ of type $\left(G_{2}, P\right)$, we can extend the structure group of the Cartan bundle, i.e., we can form the associated bundle $\tilde{\mathcal{G}}=\mathcal{G} \times_{P} \tilde{P}$. Then this is a principal bundle over $M$ with structure group $\tilde{P}$. We have a natural inclusion $j: \mathcal{G} \hookrightarrow \tilde{\mathcal{G}}$ mapping an element $u \in \mathcal{G}$ to the class $[(u, e)]$. Moreover, we can uniquely extend the Cartan connection $\omega$ on $\mathcal{G}$ to a Cartan connection $\tilde{\omega} \in \Omega^{1}(\tilde{\mathcal{G}}, \mathfrak{s o}(h))$ such that $j^{*} \tilde{\omega}=i^{\prime} \circ \omega$. The construction indeed defines a functor from Cartan geometries of type $\left(G_{2}, P\right)$ to Cartan geometries of type $(\tilde{G}, \tilde{P})$.

We will later need the relation between the curvatures of $\tilde{\omega}$ and $\omega$, which is discussed in the next lemma. We use the inclusion of adjoint tractor bundles $\mathcal{A} M \hookrightarrow \tilde{\mathcal{A}} M$ via

$$
\mathcal{A} M=\mathcal{G} \times_{P} \mathfrak{g} \hookrightarrow \mathcal{G} \times_{P} \mathfrak{s o}(h)=\tilde{\mathcal{G}} \times_{\tilde{P}} \mathfrak{s o}(h)=\tilde{A} M .
$$




\section{Lemma 2.}

1. The curvature form $\tilde{\Omega} \in \Omega^{2}(\tilde{\mathcal{G}}, \mathfrak{s o}(h))$ of $\tilde{\omega}$ pulls back to the curvature form $\Omega \in \Omega^{2}(\mathcal{G}, \mathfrak{g})$ of $\omega$ :

$$
j^{*}(\tilde{\Omega})=\Omega
$$

2. The factorizations $K \in \Omega^{2}(M, \mathcal{A} M)$ of $\Omega$ and $\tilde{K} \in \Omega^{2}(M, \tilde{\mathcal{A}} M)$ of $\tilde{\Omega}$ agree:

$$
\tilde{K}=K \in \Omega^{2}(M, \mathcal{A} M) .
$$

In particular, $K \in \Omega^{2}(M, \tilde{\mathcal{A}} M)$ has values in $\mathcal{A} M \subset \tilde{\mathcal{A}} M$.

Proof. Since the exterior derivative $d$ is natural, it commutes with pullbacks: $j^{*} d \tilde{\omega}=d\left(j^{*} \tilde{\omega}\right)=$ $d \omega$. Since also $j^{*}([\tilde{\omega}, \tilde{\omega}])=[\omega, \omega]$, we thus see that by definition of curvature (3) we have $j^{*} \tilde{\Omega}=\Omega$.

Now the inclusion $j: \mathcal{G} \rightarrow \tilde{\mathcal{G}}$ is a reduction of structure group from $\tilde{P}$ to $P$. Therefore, factorizing $\tilde{\Omega} \in \Omega_{\text {hor }}^{2}(\tilde{\mathcal{G}}, \mathfrak{s o}(h))^{\tilde{P}}$ to the curvature form $\tilde{K} \in \Omega^{2}(M, \tilde{\mathcal{A}} M)$ is the same as pulling back $\tilde{\Omega}$ via $j$ and then factorizing.

By Theorem 2, we can associate a canonical Cartan geometry $(\mathcal{G}, \omega)$ of type $\left(G_{2}, P\right)$ to a generic rank two distribution on a five manifold $M$. As discussed in Section 3, any Cartan geometry $(\tilde{\mathcal{G}}, \tilde{\omega})$ of type $(\tilde{G}, \tilde{P})$ determines a conformal structure on the underlying manifold $M$. Thus the above Fefferman construction shows that a generic rank two distribution $\mathcal{D}$ naturally determines a conformal class $[g]$ of metrics of signature $(2,3)$. However, a priori we do not know whether $\tilde{\omega}$ is the normal Cartan connection associated to that conformal structure (which will be important for applications); to see this requires a proof. We will give a proof based on the following result which is derived via BGG-techniques:

Proposition $3([7])$. Suppose $\mathbb{E} \subset \operatorname{ker}\left(\partial^{*}\right) \subset \Lambda^{2}(\mathfrak{g} / \mathfrak{p})^{*} \otimes \mathfrak{g}$ is a P-submodule, and consider the $G_{0}$-module $\mathbb{E}_{0}:=\mathbb{E} \cap \operatorname{ker}(\square)$. Let $(\mathcal{G} \rightarrow M, \omega)$ be a regular, normal parabolic geometry such that the harmonic curvature takes values in $\mathbb{E}_{0}$. If either $\omega$ is torsion-free or for any $\phi, \psi \in \mathbb{E}$ we have $\partial^{*}\left(i_{\phi} \psi\right) \in \mathbb{E}$, where $i_{\phi} \psi$ is the alternation of the map $\left(X_{0}, X_{1}, X_{2}\right) \mapsto \phi\left(\psi\left(X_{0}, X_{1}\right)+\mathfrak{p}, X_{2}\right)$ for $X_{i} \in \mathfrak{g} / \mathfrak{p}$, the curvature function $\kappa$ has takes in $\mathbb{E}$.

Kostant's version of the Bott-Borel-Weil theorem [31] provides an algorithmic description of the $G_{0}$-representation ker $(\square)$. Doing the necessary calculations for the $|3|$-graded Lie algebra $\mathfrak{g}$ of $G_{2}$ from Section 4.2 (or consulting [40], if you want the computer to calculate for you) leads to:

Lemma 3. The harmonic component $\kappa_{H}$ of a regular, normal parabolic geometry of type $\left(G_{2}, P\right)$ takes values in a $G_{0}=G_{+}(2, \mathbb{R})$-submodule of $\mathfrak{g}_{1} \wedge \mathfrak{g}_{3} \otimes \mathfrak{g}_{0}$ isomorphic to $S^{4}\left(\mathbb{R}^{2}\right)^{*}$. It follows, that the geometry is torsion-free.

Proof. An algorithmic calculation shows that the $G_{0}=\mathrm{GL}_{+}(2, \mathbb{R})$-representation $\operatorname{ker}(\square)$ is an irreducible summand of $\mathfrak{g}_{1} \wedge \mathfrak{g}_{3} \otimes \mathfrak{g}_{0}$ isomorphic to $S^{4}\left(\mathbb{R}^{2}\right)^{*}$. Since $\kappa_{H}$ takes values in $\operatorname{ker}(\square)$, it is, in particular, contained in $\Lambda^{2} \mathfrak{p}_{+} \otimes \mathfrak{p}$. Now we can apply Proposition 3 to conclude that the entire curvature $\kappa$ takes values in that $P$-module, i.e., the geometry is torsion-free.

We now show that normality of $\omega$ implies normality of $\tilde{\omega}$ :

Proposition 4. Let $(\mathcal{G} \rightarrow M, \omega)$ be a regular normal parabolic geometry of type $\left(G_{2}, P\right)$, and let $(\tilde{\mathcal{G}} \rightarrow M, \tilde{\omega})$ be the associated parabolic geometry of type $(\tilde{G}, \tilde{P})$. Then $\tilde{\omega} \in \Omega^{1}(\tilde{\mathcal{G}}, \mathfrak{s o}(h))$ is normal. 
Proof. The Killing forms of $\mathfrak{g}$ resp. $\mathfrak{s o}(h)$ provide natural identifications $\mathfrak{p}_{+} \cong(\mathfrak{g} / \mathfrak{p})^{*}$ and $\tilde{\mathfrak{p}}_{+} \cong(\mathfrak{s o}(h) / \tilde{\mathfrak{p}})^{*}$. This allows us to view the curvature function of the geometry $(\mathcal{G}, \omega)$ as a function $\kappa: \mathcal{G} \rightarrow \Lambda^{2} \mathfrak{p}_{+} \otimes \mathfrak{g}$ and the curvature function of $(\tilde{\mathcal{G}}, \tilde{\omega})$ as a function $\tilde{\kappa}: \tilde{\mathcal{G}} \rightarrow$ $\Lambda^{2} \tilde{\mathfrak{p}}_{+} \otimes \mathfrak{s o}(h)$. The inclusion $i^{\prime}: \mathfrak{g} \rightarrow \mathfrak{s o}(h)$ and the isomorphism $\mathfrak{g} / \mathfrak{p} \cong \mathfrak{s o}(h) / \tilde{\mathfrak{p}}$ induce a map $I: \Lambda^{2} \mathfrak{p}_{+} \otimes \mathfrak{g} \rightarrow \Lambda^{2} \tilde{\mathfrak{p}}_{+} \otimes \mathfrak{s o}(h)$. Since all maps involved in the construction are homomorphisms of $P$-modules, this is indeed a homomorphism of $P$-modules. In terms of $I$, the curvature functions are related via

$$
\tilde{\kappa}(j(u))=I \circ \kappa(u)
$$

for all $u \in \mathcal{G}$. By equivariance, this uniquely determines $\tilde{\kappa}$ on $\tilde{\mathcal{G}}$.

Let $\tilde{\partial}^{*}: \Lambda^{2} \tilde{\mathfrak{p}}_{+} \otimes \mathfrak{s o}(h) \rightarrow \tilde{\mathfrak{p}}_{+} \otimes \mathfrak{s o}(h)$ be the Kostant codifferential describing the conformal normalization condition, i.e., on decomposable elements $U \wedge V \otimes A \in \Lambda^{2} \tilde{\mathfrak{p}}_{+} \otimes \mathfrak{s o}(h)$ we have

$$
\tilde{\partial}^{*}(U \wedge V \otimes A)=U \otimes[V, A]-V \otimes[U, A] .
$$

To prove that the geometry $(\tilde{\mathcal{G}}, \tilde{\omega})$ is normal amounts to showing that $\tilde{\kappa}$ takes values in $\operatorname{ker}\left(\tilde{\partial}^{*}\right)$. By (28), this is equivalent to the fact that $\kappa$ takes values in $\operatorname{ker}\left(\tilde{\partial}^{*} \circ I\right)$. Proposition 3 allows to reduce this remaining problem to a purely algebraic one: Since, by Lemma 3, regular, normal parabolic geometries of type $\left(G_{2}, P\right)$ are torsion-free, Proposition 3 implies that $\kappa$ takes values in $\operatorname{ker}\left(\tilde{\partial}^{*} \circ I\right)$ if and only if this is true for the harmonic curvature component $\kappa_{H}$. By equivariance, the map $\tilde{\partial}^{*} \circ I$ either vanishes on $G_{0}$-irreducible components, or it is an isomorphism. We have observed that $\operatorname{ker}(\square)$ is an irreducible $G_{0}$-representation isomorphic to $S^{4}\left(\mathbb{R}^{2}\right)^{*}$. Hence if we can show that the image of the map $\tilde{\partial}^{*} \circ I: \Lambda^{2} \mathfrak{p}_{+} \otimes \mathfrak{g}_{0} \rightarrow \tilde{\mathfrak{p}}_{+} \otimes \mathfrak{s o}(h)$ does not contain an irreducible summand isomorphic to $S^{4}\left(\mathbb{R}^{2}\right)^{*}$, then $\kappa_{H}$ has to be contained in that kernel. But looking at formula (29), we see that $\tilde{\partial}^{*} \circ I\left(\mathfrak{p}_{+} \otimes \mathfrak{g}_{0}\right)$ is actually contained in $\tilde{\mathfrak{p}}_{+} \otimes \tilde{\mathfrak{p}}_{+}$, which is easily seen to contain no summand isomorphic to $S^{4}\left(\mathbb{R}^{2}\right)^{*}$.

\subsection{The parallel tractor three-form and the underlying conformal Killing 2-form}

Let $\mathcal{T}$ be the standard tractor bundle for a conformal structure $[g]$ associated to a generic 2-distribution $\mathcal{D}$ on a 5 -manifold $M$. Then $\mathcal{T}$ is easily seen to carry additional structure:

\section{Proposition 5.}

1. The standard tractor bundle $\mathcal{T}$ for a conformal structure $[g]$ associated to a generic 2distribution carries a parallel tractor 3 -form $\boldsymbol{\Phi} \in \Gamma\left(\Lambda^{3} \mathcal{T}^{*}\right)=\Gamma\left(\Lambda^{3} \mathcal{T}\right)$.

2. The tractor 3-form $\boldsymbol{\Phi}$ determines an underlying normal conformal Killing 2-form $\phi \in$ $\Gamma\left(\Lambda^{2} T^{*} M \otimes \mathcal{E}[3]\right)=\mathcal{E}_{[a b]}[3]$, which is locally decomposable.

Proof. 1. By construction, the conformal Cartan bundle is the associated bundle $\tilde{\mathcal{G}}=\mathcal{G} \times{ }_{P} \tilde{P}$, where $\mathcal{G}$ is the Cartan bundle for the distribution. Hence the tractor bundle can be viewed as $\mathcal{T}=\mathcal{G} \times{ }_{P} \mathbb{R}^{7}$. It follows that the $P$-equivariant function $f_{\Phi}: \mathcal{G} \rightarrow \Lambda^{3} \mathbb{R}^{7}$ mapping constantly onto the three-form $\Phi$ stabilized by $G_{2}$ induces a section $\Phi \in \Gamma\left(\Lambda^{3} \mathcal{T}\right)$. Proposition 4 implies that the normal tractor connection $\nabla^{\Lambda^{3} \mathcal{T}}$ is induced from the normal Cartan connection $\omega \in \Omega^{1}(\mathcal{G}, \mathfrak{g})$. Hence, according to (4), $\nabla_{\xi}^{\Lambda^{3} \mathcal{T}} \Phi$ corresponds to the function $u \mapsto\left(\xi_{u}^{\prime} \cdot f_{\Phi}\right)+\omega_{u}\left(\xi^{\prime}(u)\right)\left(f_{\Phi}(u)\right)$, where $u \in \mathcal{G}$ and $\xi^{\prime} \in \mathfrak{X}(\mathcal{G})$ is a $P$-invariant lift of a the vector field $\xi \in \mathfrak{X}(M)$. Since $f_{\Phi}$ is constant and $\omega$ takes values in the isotropy algebra $\mathfrak{g}$ of $\Phi$, this means that $\nabla^{\Lambda^{3} \mathcal{T}} \boldsymbol{\Phi}=0$, i.e., $\boldsymbol{\Phi}$ is a parallel tractor three-form.

2. Recall from Section 3.4 that we have a natural bundle projection $\Pi_{0}: \Lambda^{3} \mathcal{T} \rightarrow \Lambda^{2} T^{*} M \otimes \mathcal{E}[3]$ and that parallel sections of $\mathcal{V}:=\Lambda^{3} \mathcal{T}$ project to normal conformal Killing 2-forms. 
Let $V=\Lambda^{3} \mathbb{R}^{7}$ be endowed with the canonical $\tilde{P}$-invariant filtration discussed in Section 3.2. We see from our explicit formula (23) for $\Phi$ that, up to a factor, a representative in $V / V^{0}=$ $\mathrm{gr}_{-1}(V)$ is given by $e_{7} \wedge e_{2} \wedge e_{3}$. Around every point $x \in M$ we can choose a local section $\sigma: U \rightarrow \mathcal{G}$. On $\sigma(U) \subset \mathcal{G} \subset \tilde{\mathcal{G}}$ we define 3 constant functions, mapping to $e_{7}, e_{2}$ and $e_{3}$; these correspond to sections $s_{7}, s_{2}$ and $s_{3}$ of the standard tractor bundle $\mathcal{T}$. $s_{7}$ is simply $\tau_{-}$; to be precise, we use that $\sigma: U \rightarrow \mathcal{G}$ gives in particular a trivialization of the conformal weight bundles, and we can regard $\tau_{-}$as an (unweighted) section of $\mathcal{T}$. The tractors $s_{2}$ and $s_{3}$ lie in $\mathcal{T}^{0}$ and therefore project to elements $\varphi_{2}$ and $\varphi_{3}$ in $\mathcal{T}^{0} / \mathcal{T}^{1}=\operatorname{gr}_{0}(\mathcal{T})=\mathcal{E}_{a}$, where we again use the trivialization of the conformal weight bundles. Thus, $\tau_{-} \wedge \varphi_{2} \wedge \varphi_{3}$ is a representative of $\varphi=\Pi_{0}(\boldsymbol{\Phi}) \in \mathcal{V} / \mathcal{V}^{0}=\mathrm{gr}_{-1}(\mathcal{V})$, and the identification $(13)$ of $\mathcal{V} / \mathcal{V}^{0}=\mathrm{gr}_{-1}(\mathcal{V})$ with $\mathcal{E}_{[a b]}$ tells us that $\varphi=\varphi_{2} \wedge \varphi_{3} \in \mathcal{E}_{[a b]}$.

Remark 1. The parabolic subgroup $P$ of $G_{2}$ preserves a finer filtration of the standard representation, which yields the following refinement of the 'conformal' filtration of standard tractor bundle:

$$
\mathcal{T}^{2^{\prime}} \subset \mathcal{T}^{1^{\prime}} \subset \mathcal{T}^{0^{\prime}} \subset \mathcal{T}^{-1^{\prime}} \subset \mathcal{T}^{-2^{\prime}} .
$$

The isotropic line bundle $\mathcal{T}^{2^{\prime}}$ corresponds to the subspace generated by $e_{1} \in \mathbb{R}^{7}$. The bundle $\mathcal{T}^{-1^{\prime}}$ is the orthogonal complement to $\mathcal{T}^{2^{\prime}}$ with respect to the tractor metric. The explicit form of the three-form $\Phi$ (see (23)) shows how to characterize the additional filtration components in terms of $\boldsymbol{\Phi}$. Let $\tau_{+} \in \Gamma(\mathcal{T} \otimes \mathcal{E}[1])$ be the canonical insertion of $\mathcal{E}[-1]$ into $\mathcal{T}^{2^{\prime}} \subset \mathcal{T}$ which was already defined in Section 3.1. Then the subbundle $\mathcal{T}^{1^{\prime}}$ can be described as the set of all tractors $s \in \Gamma(\mathcal{T})$ such that $i_{s} i_{\tau_{+}} \mathbf{\Phi}=0$. The subbundle $\mathcal{T}^{0^{\prime}}$ is the bundle orthogonal to $\mathcal{T}^{-1^{\prime}}$.

Remark 2. The distribution $\mathcal{D}$ can be recovered from the conformal class $[g]$ associated to the distribution and the conformal Killing 2-form $\phi$. The kernel of the 2 -form is the rank three distribution $[\mathcal{D}, \mathcal{D}]$. The rank two distribution can be recovered as the kernel of the restriction of a metric $g$ to the rank three distribution, in other words, the set of isotropic elements in the kernel of $\phi$.

\section{Holonomy reduction and characterization}

The aim of the following section is to characterize conformal structures arising from generic rank 2 distributions in dimension five in terms of normal conformal Killing 2-forms satisfying certain additional equations. See Theorem A for a precise statement of the result. We proceed as follows. First, we prove that a conformal manifold of signature $(2,3)$ whose conformal holonomy is contained in $G_{2}$ is obtained from a generic rank two distribution via a Fefferman construction. Then we aim for a characterization of the conformal structures in terms of underlying conformal data; we derive conditions to distinguish those normal conformal Killing 2-forms coming from parallel tractor 3 -forms defining holonomy reductions to $G_{2}$. This is done analogously to [12], where the authors arrive at a version of Sparling's characterization [26] of Fefferman spaces in terms of a conformal Killing field.

Remark 3. Recently T. Leistner and P. Nurowski showed on examples that in some cases the conformal structures constructed in this way have explicit ambient metrics with holonomy group $G_{2}$, see [37] and [32].

\subsection{Conformal holonomy}

Let $(M,[g])$ be a conformal structure of signature $(2,3)$ encoded in a Cartan geometry $(\tilde{\mathcal{G}}, \tilde{\omega})$ as described in Section 3. The standard tractor bundle $\mathcal{T}$ of $[g]$ is endowed with the tractor 
connection $\nabla^{\mathcal{T}}$, and we define the conformal holonomy of $[g]$ as

$$
\operatorname{Hol}([g]):=\operatorname{Hol}\left(\nabla^{\mathcal{T}}\right) .
$$

See also [2]. Now $\mathcal{T}$ comes about as associated bundle to $\tilde{\mathcal{G}}^{\prime}:=\tilde{\mathcal{G}} \times \tilde{P} \mathrm{SO}(h)$ and $\nabla^{\mathcal{T}}$ is induced from the principal connection form $\tilde{\omega}^{\prime} \in \Omega^{1}\left(\tilde{\mathcal{G}}^{\prime}, \mathfrak{s o}(h)\right)$. Thus, we have that $\operatorname{Hol}\left(\nabla^{\mathcal{T}}\right)=\operatorname{Hol}\left(\tilde{\omega}^{\prime}\right)$. By construction, the pullback of $\tilde{\omega}^{\prime}$ to $\tilde{\mathcal{G}} \subset \tilde{\mathcal{G}}^{\prime}$ is the Cartan connection form $\tilde{\omega}$.

In the Fefferman-type construction of Section 4.4 we started with a parabolic geometry $(\mathcal{G}, \omega)$ of type $\left(G_{2}, P\right)$ encoding a generic rank 2 distribution $\mathcal{D}$, and we associated to this the parabolic geometry $(\tilde{\mathcal{G}}, \tilde{\omega})$ of type $(\mathrm{SO}(h), \tilde{P})$ by equivariantly extending $\omega$ to $\tilde{\omega}$. If we add the extended bundles $\mathcal{G}^{\prime}=\mathcal{G} \times{ }_{P} G_{2}$ and $\tilde{\mathcal{G}}^{\prime}=\tilde{\mathcal{G}} \times{ }_{\tilde{P}} \mathrm{SO}(h)=\mathcal{G} \times{ }_{P} \mathrm{SO}(h)$ to the picture, we obtain the commuting diagram of inclusions

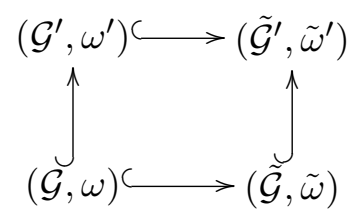

In particular, this yields a holonomy reduction of $\left(\tilde{\mathcal{G}}^{\prime}, \tilde{\omega}^{\prime}\right)$ to $\left(\mathcal{G}^{\prime}, \omega^{\prime}\right)$, and thus $\operatorname{Hol}\left(\tilde{\omega}^{\prime}\right)=$ $\operatorname{Hol}\left(\omega^{\prime}\right) \subset G_{2}$. By Proposition 4, $\tilde{\omega}$ is normal. Hence, $\operatorname{Hol}\left(\tilde{\omega}^{\prime}\right)$ is indeed the conformal holonomy $\operatorname{Hol}\left([g]_{\mathcal{D}}\right)$, which is thus seen to be contained in $G_{2}$.

We are now going to show the converse: if for a conformal structure $(M,[g])$ of signature $(2,3)$ the conformal holonomy $\operatorname{Hol}([g])$ is contained in $G_{2}$, then there is a canonical generic rank 2distribution $\mathcal{D}$ on $M$ such that $[g]=[g]_{\mathcal{D}}$.

Let $\pi: \tilde{\mathcal{G}}^{\prime} \rightarrow M$ be the surjective submersion of the $\mathrm{SO}(h)$-principal bundle $\tilde{\mathcal{G}}^{\prime}$. The next proposition covers the holonomy reduction of a conformal Cartan geometry to $G_{2}$. A similar result has been obtained in [1].

Proposition 6. Let $(\tilde{\mathcal{G}}, \tilde{\omega})$ be such that $\left(\tilde{\mathcal{G}}^{\prime}, \tilde{\omega}^{\prime}\right)$ has holonomy in $G_{2}$ and let $\mathcal{H} \subset \tilde{\mathcal{G}}^{\prime}$ be a reduction of $\left(\tilde{\mathcal{G}}^{\prime}, \tilde{\omega}^{\prime}\right)$ to $G_{2}$. Then:

1. $\mathcal{H} \subset \tilde{\mathcal{G}}^{\prime}$ and $\tilde{\mathcal{G}} \subset \tilde{\mathcal{G}}^{\prime}$ intersect transversally. We denote the resulting submanifold by $\mathcal{G}:=\mathcal{H} \cap \tilde{\mathcal{G}}$.

2. For every $u \in \mathcal{G}, T_{u} \pi\left(T_{u} \mathcal{G}\right)=T_{\pi(u)} M$.

3. $\mathcal{G}$ is a P-principal bundle over $M$.

4. Let $\omega$ be the pullback of $\tilde{\omega} \in \Omega^{1}(\mathcal{G}, \mathfrak{s o}(h))$ to $\mathcal{G}$. Then $\omega \in \Omega^{1}(\mathcal{G}, \mathfrak{g})$ is a Cartan connection form.

Proof. 1. We have that $T_{u} \mathcal{H}+T_{u} \tilde{\mathcal{G}} \supset u \cdot \mathfrak{g}+u \cdot \tilde{\mathfrak{p}}=u \cdot \mathfrak{s o}(h)=\operatorname{ker}\left(T_{u} \pi\right)$. Since $T_{u} \pi: T_{u} \tilde{\mathcal{G}} \rightarrow$ $T_{\pi(u)} M$ is surjective, we have that $\operatorname{dim}\left(T_{u} \mathcal{H}+T_{u} \tilde{\mathcal{G}}\right)=\operatorname{dim}(\mathfrak{s o}(h))+\operatorname{dim}(\mathfrak{g} / \mathfrak{p})=\operatorname{dim}\left(T_{u} \tilde{\mathcal{G}}^{\prime}\right)$.

2. Take $u \in \mathcal{G}=\mathcal{H} \cap \tilde{\mathcal{G}}$ and $\xi \in T_{\pi(u)} M$. Since the restrictions of $\pi$ to $\mathcal{H}$ and $\tilde{\mathcal{G}}$ are surjective submersions, there exist $\xi_{1} \in T_{u} \mathcal{H}$ and $\xi_{2} \in T_{u} \tilde{\mathcal{G}}$ such that $\xi=T_{u} \pi \xi_{1}=T_{u} \pi \xi_{2}$. Then

$$
\xi_{1}-\xi_{2} \in \operatorname{ker} T_{u} \pi=u \cdot \mathfrak{s o}(h)=u \cdot \mathfrak{g}+u \cdot \tilde{\mathfrak{p}} .
$$

Thus, there exist $\eta_{1} \in u \cdot \mathfrak{g}$ and $\eta_{2} \in u \cdot \tilde{\mathfrak{p}}$ such that $\xi_{1}-\xi_{2}=\eta_{1}+\eta_{2}$. Let

$$
\xi^{\prime}=\xi_{1}-\eta_{1}=\xi_{2}+\eta_{2} \in T_{u} \mathcal{G} .
$$

Then indeed $T_{u} \pi \xi^{\prime}=\xi$.

3. Assume first that for an $x \in M$ there is a $u \in \mathcal{H}_{x} \cap \tilde{\mathcal{G}}_{x}=\mathcal{G}_{x}$. Then evidently $\mathcal{G}_{x}=$ $u \cdot\left(G_{2} \cap \tilde{P}\right)=u \cdot P$. It therefore remains to show that $\mathcal{H}_{x} \cap \tilde{\mathcal{G}}_{x}$ is always non-empty: let $u \in \mathcal{H}_{x}$. 
Then there is a $g \in \mathrm{SO}(h)$ such that $u \cdot g \in \tilde{\mathcal{G}}$. Since $G_{2} / P=\operatorname{SO}(h) / \tilde{P}$ (see Section 4.2), there is a $p \in \tilde{\mathcal{P}}$ such that $g p=g^{\prime} \in G_{2}$, and then $u \cdot g^{\prime} \in \mathcal{H}$ since $\mathcal{H}$ is a $G_{2}$-subbundle and $u \cdot g^{\prime}=(u \cdot g) \cdot p \in \mathrm{SO}(h)$; i.e., $u \cdot g^{\prime} \in \mathcal{G}$.

4. We now consider $\mathcal{G}$ as a reduction of the $\tilde{P}$-principal bundle $\tilde{\mathcal{G}}$ to $P$ and denote by $\omega$ the pullback of $\tilde{\omega} \in \Omega^{1}(\tilde{\mathcal{G}}, \mathfrak{s o}(h))$. By construction, $\mathcal{H} \subset \mathcal{G}$ was obtained by holonomy reduction of $\left(\tilde{\mathcal{G}}^{\prime}, \tilde{\omega}^{\prime}\right)$ to $G_{2}$. In particular, $\tilde{\omega}_{\mid T \mathcal{H}}^{\prime}$ has values in $\mathfrak{g}$, and thus $\omega \in \Omega^{1}(\mathcal{G}, \mathfrak{g})$. P-equivariance and reproduction of $\mathfrak{p}$-fundamental vector fields is clear since $\mathcal{G}$ is just a $P$-principal subbundle of $\tilde{\mathcal{G}}$ and $\tilde{\omega}$ is a Cartan connection form satisfying (C.1)-(C.2) by assumption. We thus need to check that also (C.3) holds for $\omega$. I.e., for every $u \in \mathcal{G}$ we need that $\omega_{u}: T_{u} \mathcal{G} \rightarrow \mathfrak{g}$ is an isomorphism. We have seen that $T_{u} \pi\left(T_{u} \mathcal{G}\right)=T_{\pi(u)} M$. Since $u \cdot \tilde{\mathfrak{p}}=\operatorname{ker}\left(T_{u} \pi\right) \subset T_{u} \tilde{\mathcal{G}}$ we see that $T_{u} \mathcal{G} \subset T_{u} \tilde{\mathcal{G}}$ must span at least $\operatorname{dim}(\mathfrak{g} / \mathfrak{p})$-complementary dimensions and thus already $T_{u} \mathcal{G}+u \cdot \mathfrak{p}=T_{u} \tilde{\mathcal{G}}$. But then $\omega_{u}\left(T_{u} \mathcal{G} / u \cdot \mathfrak{p}\right)=\tilde{\omega}_{u}\left(T_{u} \tilde{\mathfrak{g}} / u \cdot \tilde{\mathfrak{p}}\right)=\tilde{\mathfrak{g}} / \tilde{\mathfrak{p}}=\mathfrak{g} / \mathfrak{p}$. This, together with $\omega_{u}(u \cdot \mathfrak{p})=\mathfrak{p}$ by reproduction of fundamental vector fields, gives that indeed $\omega_{u}\left(T_{u} \mathcal{G}\right)=\mathfrak{g}$.

Now suppose $(\tilde{\mathcal{G}}, \tilde{\omega})$ is a normal parabolic geometry of type $(\tilde{G}, \tilde{P})$ associated to $[g]$ with $\operatorname{Hol}([g]) \subset G_{2}$, and let $(\mathcal{G}, \omega)$ be the parabolic geometry of type $\left(G_{2}, P\right)$ obtained via reduction as explained in Proposition 6. Since every normal conformal Cartan connection is torsion-free and $\mathfrak{p} \subset \tilde{\mathfrak{p}}, \omega$ is torsion-free. This evidently implies that $\omega$ is regular, and therefore it determines an underlying generic 2-distribution. We will show in Theorem 3 that the canonical conformal structure associated to this distribution is $[g]$; this will employ the following

Lemma 4. Let $(\tilde{\mathcal{G}}, \tilde{\omega})$ be a normal parabolic geometry of type $(\tilde{G}, \tilde{P})$ with $\operatorname{Hol}\left(\tilde{\omega}^{\prime}\right) \subset G_{2}$, and let $(\mathcal{G}, \omega)$ be the parabolic geometry of type $\left(G_{2}, P\right)$ obtained via reduction. Then there is a normal Cartan connection $\omega_{N} \in \Omega^{1}(\mathcal{G}, \mathfrak{g})$ such that the difference $\left(\omega_{N}-\omega\right)$ is of homogeneous degree $\geq 3$.

Proof. Let $\kappa: \mathcal{G} \rightarrow \Lambda^{2} \mathfrak{p}_{+} \otimes \mathfrak{g}$ be the curvature function of $\omega$. We will verify that $\kappa$ is of homogeneous degree $\geq 3$. Since the Kostant codifferential preserves homogeneities, then also $\partial^{*} \kappa$ is of homogeneous degree $\geq 3$, and by Proposition 2 this shows that there is a normal Cartan connection $\omega_{N}$ that differs from $\omega$ at most in homogeneous degree $\geq 3$.

Torsion-freeness of $\omega$ means that for any $u \in \mathcal{G}, \kappa(u)$ is contained in $\Lambda^{2} \mathfrak{p}_{+} \otimes \mathfrak{p}$. Hence the only component of $\kappa(u)$ of homogeneous degree $<3$ that remains to be investigated is

$$
\kappa_{2}(u) \in \mathfrak{g}_{1} \otimes \mathfrak{g}_{1} \otimes \mathfrak{g}_{0} .
$$

We show that this component vanishes as well.

Let $\tilde{\partial}^{*}: \Lambda^{2} \tilde{\mathfrak{p}}_{+} \otimes \mathfrak{s o}(h) \rightarrow \tilde{\mathfrak{p}}_{+} \otimes \mathfrak{s o}(h)$ be the conformal Kostant codifferential. Choose linearly independent elements $X_{1}, X_{2} \in \mathfrak{g}_{-1}, X_{3} \in \mathfrak{g}_{-2}$ and $X_{4}, X_{5} \in \mathfrak{g}_{-3}$; then these elements give a basis of $\mathfrak{s o}(h) / \tilde{\mathfrak{p}}$. Consider the dual basis $\tilde{Z}_{1}, \ldots, \tilde{Z}_{5} \in \tilde{\mathfrak{p}}_{+}$with respect to the Killing form on $\mathfrak{s o}(h)$. By construction, $\kappa(u)\left(X_{i}, X_{j}\right)=\tilde{\kappa}(u)\left(X_{i}, X_{j}\right)$ for all $u \in \mathcal{G}$. Thus normality of the conformal Cartan connection implies

$$
\sum_{i<j}\left(\left[\tilde{Z}_{i}, \kappa(u)\left(X_{i}, X_{j}\right)\right] \otimes \tilde{Z}_{j}-\left[\tilde{Z}_{j}, \kappa(u)\left(X_{i}, X_{j}\right)\right] \otimes \tilde{Z}_{i}\right)=0 .
$$

Recall that we have a $\mathfrak{g}$-module decomposition $\mathfrak{s o}(h)=\mathfrak{g} \oplus \mathbb{V}$. The projection $\pi_{\mathfrak{g}}: \mathfrak{s o}(h) \rightarrow \mathfrak{g}$ maps $\tilde{Z}_{j}$ to an element $Z_{j} \in \mathfrak{p}_{+}$dual to $X_{j} \in \mathfrak{g}_{-}$with respect to a multiple of the Killing form on $\mathfrak{g}$. Equivariance of the projection implies $\pi_{\mathfrak{g}}\left(\left[\tilde{Z}_{i}, \kappa(u)\left(X_{i}, X_{j}\right)\right]\right)=\left[Z_{i}, \kappa(u)\left(X_{i}, X_{j}\right)\right]$. It follows that (30) also holds with $\tilde{Z}_{i}$ 's replaced by $Z_{i}$ 's. The only part of that sum contained in lowest homogeneity, i.e. in $\mathfrak{g}_{1} \otimes \mathfrak{g}_{1}$, is

$$
\left[Z_{1}, \kappa_{2}(u)\left(X_{1}, X_{2}\right)\right] \otimes Z_{2}-\left[Z_{2}, \kappa_{2}(u)\left(X_{1}, X_{2}\right)\right] \otimes Z_{1},
$$

and so this expression vanishes as well. Since the representation of $\mathfrak{g}_{0}$ on $\mathfrak{g}_{1}$ given by the Lie bracket is faithful, this indeed implies that $\kappa_{2}(u)\left(X_{1}, X_{2}\right)=0$. 
Having Proposition 6 and Lemma 4, we can now show:

Theorem 3. Let $(M,[g])$ be a conformal structure of signature $(2,3)$ with conformal holonomy $\operatorname{Hol}([g]) \subset G_{2}$. Then $[g]$ is canonically associated to a generic rank two distribution $\mathcal{D}$ via a Fefferman-type construction.

Proof. Let $(\tilde{\mathcal{G}}, \tilde{\omega})$ be the normal parabolic geometry of type $(\mathrm{SO}(h), \tilde{P})$ associated to the conformal structure $[g]$. Let $(\mathcal{G}, \omega)$ be the parabolic geometry of type $\left(G_{2}, P\right)$ constructed in Proposition 6 . Then we know that $\omega$ is regular, and by Lemma 4 there is a normal Cartan connection $\omega_{N} \in \Omega^{1}(\mathcal{G}, \mathfrak{g})$ that differs from $\omega$ at most in homogeneous degree $\geq 3$.

Recall that the Cartan connection $\omega$ determines an isomorphism $\mathcal{G} \times_{P} \mathfrak{g} / \mathfrak{p} \cong T M$. Regularity of $\omega$ implies that the image of $\mathcal{G} \times_{P} \mathfrak{g}^{-1} / \mathfrak{p}$ under this isomorphism is a generic rank two distribution $\mathcal{D}$. Furthermore, we have a $P$-invariant conformal class of bilinear forms of signature $(2,3)$ on $\mathfrak{g} / \mathfrak{p}$, and the conformal structure induced via the above isomorphism on $M$ is just $[g]$. On the other hand, the Fefferman construction associates a conformal structure $[g]_{\mathcal{D}}$ to the distribution $\mathcal{D}$. This is the conformal structure induced via the isomorphism $\mathcal{G} \times_{P} \mathfrak{g} / \mathfrak{p} \cong T M$ defined by the normal Cartan connection $\omega_{N} \in \Omega^{1}(\mathcal{G}, \mathfrak{g})$ associated to the distribution $\mathcal{D}$. Since $\omega_{N}-\omega$ is of homogeneous degree $\geq 3$, the difference $\left(\omega-\omega_{N}\right)$ takes values in $\mathfrak{p}$. But this implies that $\omega$ and $\omega_{N}$ induce the same isomorphism $T M \cong \mathcal{G} \times{ }_{P} \mathfrak{g} / \mathfrak{p}$ and hence the same conformal structure on $M$; i.e., the conformal structure $[g]$ is the one induced by the distribution $\mathcal{D}:[g]=[g]_{\mathcal{D}}$.

\subsection{Characterization via the tractor 3 -form}

We have seen that conformal structures associated to generic rank two distributions in dimension five precisely correspond to reductions in conformal holonomy from $\mathrm{SO}(h)$ to $G_{2}$. As a next step towards the desired characterization result, we explain that such a holonomy reduction can be encoded in terms of a parallel tractor 3-form satisfying a certain compatibility condition with the tractor metric.

Let $\tilde{\mathcal{G}}^{\prime}$ be the extended $\mathrm{SO}(h)$-principal bundle over $M$, and let $\tilde{\omega}^{\prime} \in \Omega^{1}\left(\tilde{\mathcal{G}}^{\prime}, \mathfrak{s o}(h)\right)$ be the extension of the Cartan connection to a principal connection form. We consider the holonomy group $\operatorname{Hol}_{u}=\operatorname{Hol}_{u}\left(\tilde{\omega}^{\prime}\right)$ for an arbitrary point $u \in \tilde{\mathcal{G}}^{\prime}$. Then, for $g \in \mathrm{SO}(h)$, one has $\mathrm{Hol}_{u \cdot g}=$ $g \mathrm{Hol}_{u} g^{-1}$, and $\operatorname{Hol}([g])$ is well defined up to conjugation in $\mathrm{SO}(h)$.

Let $\mathcal{H}_{u} \hookrightarrow \tilde{\mathcal{G}}^{\prime}, u \in \tilde{\mathcal{G}}^{\prime}$, be the reduction of the $\mathrm{SO}(h)$-bundle $\tilde{\mathcal{G}}^{\prime}$ to $\operatorname{Hol}_{u}$. If $\boldsymbol{\Psi} \in \Lambda^{3} \mathcal{T}$ is parallel, it corresponds to a $\mathrm{SO}(h)$-equivariant function $f: \tilde{\mathcal{G}}^{\prime} \rightarrow \Lambda^{3} \mathbb{R}^{7}$ which is a constant $\Psi_{u} \in \Lambda^{3} \mathbb{R}^{7}$ on $\mathcal{H}_{u}$. Hence $\mathrm{Hol}_{u} \cdot \Psi_{u}=\Psi_{u}$, or $\operatorname{Hol}_{u} \subset \mathrm{SO}(h)_{\Psi_{u}}$. If $u^{\prime}$ is another point in $\tilde{\mathcal{G}}^{\prime}$ one has $\operatorname{Hol}_{u^{\prime}}=g \operatorname{Hol}_{u} g^{-1}$ for some $g \in \mathrm{SO}(h)$ and $\Psi_{u^{\prime}}=g \cdot \Psi_{u}$. Thus $f\left(\tilde{\mathcal{G}}^{\prime}\right)=\mathrm{SO}(h) \cdot \Psi_{u}$. We say that $\mathrm{SO}(h)_{\Psi_{u}}$ is the orbit type of the parallel tractor $\boldsymbol{\Psi}$. To be precise, the orbit type is defined up to conjugation in $\mathrm{SO}(h)$.

Recall that the group $G_{2} \subset \mathrm{SO}(h)$ has been realized as the stabilizer $\mathrm{SO}(h)_{\Phi}$ for $\Phi \in \Lambda^{3} \mathbb{R}^{7}$ given by (23). Compatibility condition (24) singles out the $\operatorname{SO}(h)$-orbit of $\Phi \in \Lambda^{3} \mathbb{R}^{7}$. Hence $\operatorname{Hol}([g])$ reduces to $G_{2}$ if and only if there is a $\nabla^{\Lambda^{3} \mathcal{T}}$-parallel $\Phi \in \Lambda^{3} \mathcal{T}$ satisfying the global version of (24), i.e.,

$$
H(\boldsymbol{\Phi})=\lambda \mathbf{h} \text { for a } \lambda \in \mathbb{R} \backslash\{0\}
$$

where, for $s_{1}, s_{2} \in \Gamma(\mathcal{T})$,

$$
H(\mathbf{\Phi})\left(s_{1}, s_{2}\right) \mathbf{v o l}=i_{s_{1}} \mathbf{\Phi} \wedge i_{s_{2}} \mathbf{\Phi} \wedge \mathbf{\Phi},
$$

and vol $\in \Lambda^{7} \mathcal{T}^{*}$ is the tractor volume form. 


\subsection{Characterization in terms of the underlying conformal Killing 2-form}

We want to express compatibility condition (31) of the $\nabla^{\Lambda^{3} \mathcal{T}}$-parallel tractor $\boldsymbol{\Phi} \in \Lambda^{3} \mathcal{T}$ in terms of the underlying normal conformal Killing 2-form $\phi=\Pi_{0}(\boldsymbol{\Phi}) \in \Gamma\left(\Lambda^{2} T^{*} M \otimes \mathcal{E}[3]\right)$.

According to (19) we have

$$
\left.\boldsymbol{\Phi}=\left(\begin{array}{c}
\rho_{a_{1} a_{2}} \\
\varphi_{a_{0} a_{1} a_{2}} \mid \mu_{a_{2}} \\
\phi_{a_{1} a_{2}}
\end{array}\right)=\left(\begin{array}{c}
-\frac{1}{15} D^{p} D_{p} \phi_{a_{1} a_{2}}+\frac{2}{15} D^{p} D_{\left[a_{1}\right.} \phi_{\left.|p| a_{2}\right]}+\frac{1}{10} D_{\left[a_{1}\right.} D^{p} \phi_{\left.|p| a_{2}\right]} \\
+\frac{4}{5} P_{\left[a_{1}\right.}^{p} \phi_{\left.|p| a_{2}\right]}-\frac{1}{5} J \phi_{a_{1} a_{2}} \\
D_{\left[a_{0}\right.} \phi_{\left.a_{1} a_{2}\right]} \mid-\frac{1}{4} \boldsymbol{g}^{p q} D_{p} \phi_{q a_{2}} \\
\phi_{a_{1} a_{2}}
\end{array}\right)\right) .
$$

We will also write this splitting as

$$
\Phi=\tau_{-} \wedge \phi+\varphi+\tau_{+} \wedge \tau_{-} \wedge \mu+\tau_{+} \wedge \rho=\left(\begin{array}{c}
\rho \\
\varphi \mid \mu \\
\phi
\end{array}\right) .
$$

By (20) the conditions for a conformal Killing 2-form $\phi \in \Omega^{2}(M) \otimes \mathcal{E}[3]$ to be normal are

$$
\begin{aligned}
& D_{c} \rho_{a_{1} a_{2}}-P_{c}^{p} \varphi_{p a_{1} a_{2}}-2 P_{c\left[a_{1}\right.} \mu_{\left.a_{2}\right]}=0, \\
& D_{c} \varphi_{a_{0} a_{1} a_{2}}+3 \boldsymbol{g}_{c\left[a_{0}\right.} \rho_{\left.a_{1} a_{2}\right]}+3 P_{c\left[a_{0}\right.} \phi_{\left.a_{1} a_{2}\right]}=0, \\
& D_{c} \mu_{a_{2}}-P_{c}^{p} \phi_{p a_{2}}+\rho_{c a_{2}}=0 .
\end{aligned}
$$

We now consider the map $H: \Lambda^{3} \mathcal{T} \rightarrow S^{2} \mathcal{T}^{*}$ defined in (32). As a $\mathrm{SO}(h)$-representation $S^{2} \mathbb{R}^{7^{*}}$ decomposes into the irreducible components $S_{0}^{2} \mathbb{R}^{7^{*}}$ of trace-free symmetric 2-forms and the space $\mathbb{R} h$ of multiples of $h$. The corresponding decomposition on the tractor level is

$$
S^{2} \mathcal{T}^{*}=S_{0}^{2} \mathcal{T}^{*} \oplus \mathbb{R} \mathbf{h} .
$$

Accordingly, $H(\boldsymbol{\Phi})$ decomposes into $H(\boldsymbol{\Phi})_{0}$ and $H(\boldsymbol{\Phi})_{t r}$. Compatibility condition (31) then means that $H(\boldsymbol{\Phi})_{0}=0$ and $H(\boldsymbol{\Phi})_{t r} \neq 0$.

Lemma 5. A parallel tractor 3 -form $\Phi$ satisfies $H(\mathbf{\Phi})_{0}=0$ if and only if

$$
\phi \wedge \phi \wedge \mu=0 .
$$

In particular, $H(\boldsymbol{\Phi})_{0}=0$ whenever $\phi$ is locally decomposable.

Proof. $S_{0}^{2} \mathcal{T}^{*}$ is the tractor-bundle associated to the irreducible representation of $\mathrm{SO}(h)$ on $S_{0}^{2} \mathbb{R}^{n+2^{*}}$. By assumption, $\boldsymbol{\Phi}$ is $\nabla^{\Lambda^{3} \mathcal{T}}$-parallel. The mapping $\boldsymbol{\Phi} \mapsto H(\boldsymbol{\Phi}) \mapsto H(\boldsymbol{\Phi})_{0}$ is algebraic, and thus naturality of the tractor connection implies that $H(\boldsymbol{\Phi})_{0}$ is $\nabla^{S_{0}^{2} \mathcal{T}^{*}}$-parallel. The section $H(\boldsymbol{\Phi})_{0} \in \Gamma\left(S_{0}^{2} \mathcal{T}^{*}\right)$ can thus by Lemma 1 be recovered via the BGG-splitting operator $L_{0}^{S_{0}^{2} \mathcal{T}^{*}}$ from its projection to $\mathcal{H}_{0}\left(S_{0}^{2} \mathcal{T}^{*}\right)=\mathcal{E}[2]$. This projection is achieved by inserting twice the top slot $\tau_{+}$into $H(\boldsymbol{\Phi})_{0}$, but since $\mathbf{h}\left(\tau_{+}, \tau_{+}\right)=0$ this is the same as evaluating $H(\boldsymbol{\Phi})\left(\tau_{+}, \tau_{+}\right)$. Now according to $(32)$

$$
H(\boldsymbol{\Phi})\left(\tau_{+}, \tau_{+}\right) \operatorname{vol}=\left(i_{\tau_{+}}(\mathbf{\Phi})\right) \wedge\left(i_{\tau_{+}}(\mathbf{\Phi})\right) \wedge \boldsymbol{\Phi} .
$$

Using the representation (33) with respect to a metric $g \in[g]$, we have $i_{\tau_{+}} \mathbf{\Phi}=\phi-\tau_{+} \wedge \mu$ and thus

$$
\begin{aligned}
H(\mathbf{\Phi})\left(\tau_{+}, \tau_{+}\right) \operatorname{vol} & =\left(\phi-\tau_{+} \wedge \mu\right) \wedge\left(\phi-\tau_{+} \wedge \mu\right) \wedge\left(\tau_{-} \wedge \phi+\varphi+\tau_{+} \wedge \tau_{-} \wedge \mu+\tau_{+} \wedge \rho\right) \\
& =\phi \wedge \phi \wedge \tau_{+} \wedge \tau_{-} \wedge \mu-\tau_{+} \wedge \mu \wedge \phi \wedge \tau_{-} \wedge \phi-\phi \wedge \tau_{+} \wedge \mu \wedge \tau_{-} \wedge \phi \\
& =3 \tau_{+} \wedge \tau_{-} \wedge \phi \wedge \phi \wedge \mu .
\end{aligned}
$$

This vanishes if and only if $\phi \wedge \phi \wedge \mu=0$. 
Assume now that $H(\boldsymbol{\Phi})_{0}$ vanishes, i.e. $H(\boldsymbol{\Phi})=H(\boldsymbol{\Phi})_{t r}=\lambda \mathbf{h}$, and since $0=\nabla^{S^{2}} \mathcal{T}_{0}^{*}(\lambda \mathbf{h})=$ $(d \lambda) \mathbf{h}$ we have that $\lambda \in \mathbb{R}$ is a constant.

Lemma 6. Suppose that $H(\mathbf{\Phi})_{0}=0$. Then, $H(\boldsymbol{\Phi})=\lambda \mathbf{h}$ for a constant $\lambda \in \mathbb{R}, \lambda \neq 0$, if and only if

$$
\phi \wedge \mu \wedge \rho \neq 0 .
$$

Proof. We check that $\lambda \neq 0$ by inserting $\tau_{+}, \tau_{-}$since $H(\boldsymbol{\Phi})\left(\tau_{+}, \tau_{-}\right)=\lambda \mathbf{h}\left(\tau_{+}, \tau_{-}\right)=\lambda$ :

$$
\begin{aligned}
H(\boldsymbol{\Phi})\left(\tau_{+}, \tau_{-}\right) \mathbf{v o l} & =\left(i_{\tau_{+}} \boldsymbol{\Phi}\right) \wedge\left(i_{\tau_{-}} \boldsymbol{\Phi}\right) \wedge \boldsymbol{\Phi} \\
& =\left(\phi-\tau_{+} \wedge \mu\right) \wedge\left(\tau_{-} \wedge \mu+\rho\right) \wedge\left(\tau_{-} \wedge \phi+\varphi+\tau_{+} \wedge \tau_{-} \wedge \mu+\tau_{+} \wedge \rho\right) \\
& =\phi \wedge \tau_{-} \wedge \mu \wedge \tau_{+} \wedge \rho+\phi \wedge \rho \wedge \tau_{+} \wedge \tau_{-} \wedge \mu-\tau_{+} \wedge \mu \wedge \rho \wedge \tau_{-} \wedge \phi \\
& =3 \tau_{+} \wedge \tau_{-} \wedge \phi \wedge \mu \wedge \rho .
\end{aligned}
$$

Thus, $\lambda \neq 0$ if and only if $\phi \wedge \mu \wedge \rho \neq 0$. Note that this fixes the constant $\lambda$ and $\phi \wedge \mu \wedge \rho$ either vanishes globally or nowhere.

We are now ready to prove Theorem A:

Theorem A. Let $[g]$ be a conformal class of signature $(2,3)$ metrics on $M$. Then $[g]$ is induced from a generic rank 2 distribution $\mathcal{D} \subset T M$ if and only if there exists a normal conformal Killing 2 -form $\phi$ which is locally decomposable and satisfies the genericity condition

$$
\phi \wedge \mu \wedge \rho \neq 0 .
$$

Proof. Suppose $[g]$ is induced from a generic rank 2 distribution $\mathcal{D} \subset T M$. Then Proposition 5 shows that there is a parallel tractor 3 -form $\boldsymbol{\Phi} \in \Gamma\left(\Lambda^{3} \mathcal{T}\right)$ that projects to a locally decomposable normal conformal Killing 2-form $\phi=\Pi_{0}(\boldsymbol{\Phi})$. Furthermore, the discussion in Section 5.2 shows that $H(\boldsymbol{\Phi})$ is a nonzero multiple of the tractor metric $\mathbf{h}$. This implies $\phi \wedge \mu \wedge \rho \neq 0$ by Lemma 6 .

Conversely, suppose we have a locally decomposable normal conformal Killing 2-form $\phi \in$ $\Gamma\left(\Lambda^{2} T^{*} M \otimes \mathcal{E}[3]\right)$ satisfying the genericity condition. According to Section 3.4, $\phi$ corresponds to a parallel tractor 3 -form $\boldsymbol{\Phi}$ given by (33). Lemmata 5 and 6 show that the assumptions on $\phi$ imply $H(\boldsymbol{\Phi})=\lambda \mathbf{h}$, for $\lambda \neq 0$, and thus $\operatorname{Hol}([g]) \subset G_{2}$, as explained in Section 5.2. By Theorem 3, this means that the conformal structure is canonically associated to a 2-distribution $\mathcal{D}$.

Remark 4. It is a well known consequence of the classical Plücker relations (cf. [21]) that a two form $\varphi$ is locally decomposable if and only if $\varphi \wedge \varphi$ vanishes globally.

Remark 5. Throughout this paper we have assumed orientability of $T M$. This however, is only a minor point: If we leave this assumption and denote by $\mathcal{O}$ the 2 -fold covering of $M$ which is the orientation-bundle, we would obtain a twisted normal conformal Killing 2-form $\phi \in \Gamma\left(\Lambda^{2} T^{*} M \otimes \mathcal{E}[3] \otimes \mathcal{O}\right)$.

\section{Decomposition of conformal Killing fields of $[g]_{\mathcal{D}}$}

The goal of this section is to prove Theorem B. We will show that every conformal Killing field of $[g]_{\mathcal{D}}$ decomposes into a symmetry of the distribution $\mathcal{D}$ and an almost Einstein scale. The space of almost Einstein scales aEs $([g])$ was defined in (18) of Section 3.3. Now we discuss symmetries.

Since $\mathcal{D}$ and $[g]$ are equivalently described by Cartan geometries $(\mathcal{G}, \omega)$ resp. $(\tilde{\mathcal{G}}, \tilde{\omega})$ we can determine their symmetry algebras by determining the symmetries of their corresponding Cartan 
geometries - in fact, one can define them in this way. For this purpose, we give the general description [9] of the Lie algebra of infinitesimal automorphisms of a parabolic geometry below in Section 6.2.

Before this Cartan-geometric description, let us discuss the classical notions. An infinitesimal automorphism or symmetry of the distribution $\mathcal{D} \subset T M$ is a vector field on $M$ whose Lie derivative preserves $\mathcal{D}$, i.e.,

$$
\operatorname{sym}(\mathcal{D})=\left\{\xi \in \mathfrak{X}(M): \mathcal{L}_{\xi} \eta=[\xi, \eta] \subset \Gamma(\mathcal{D}) \forall \eta \in \Gamma(\mathcal{D})\right\} .
$$

Remark 6. In this text we won't show directly that the symmetries of the distribution $\mathbf{s y m}(\mathcal{D})$ defined via (35) agree with the infinitesimal automorphisms $\inf (\omega)$ of the corresponding Cartan geometry discussed below. We just use the fact that associating a regular normal parabolic geometry of type $\left(G_{2}, P\right)$ to a generic rank 2-distribution $\mathcal{D}$ is an equivalence of symmetries. The explicit form of the splitting from vector fields on $M$ into the adjoint tractor bundle relating the classical and the Cartan-viewpoint is only given in the conformal case, since there we will later need the explicit formula.

\subsection{Conformal Killing fields}

The symmetries of the associated conformal structure $[g]=[g]_{\mathcal{D}}$ are the conformal Killing fields

$$
\mathbf{c K f}([g])=\left\{\xi \in \mathfrak{X}(M): \mathcal{L}_{\xi} g=e^{2 f} g \text { for some } g \in[g] \text { and } f \in \mathrm{C}^{\infty}(M)\right\} .
$$

Since $\mathcal{L}_{\xi} g$ decomposes into a multiple of $g$ and a trace-free part one can equivalently demand that $\mathcal{L}_{\xi} g$ is pure trace. Now, with $D$ the Levi-Civita connection of $g \in[g], \mathcal{L}_{\xi} g$ being pure trace is equivalent to

$$
D_{(c} \xi_{a)_{0}}=D_{(c} g_{a)_{0} p} \xi^{p}=0
$$

i.e., the symmetric, trace-free part of $D_{c} \xi_{a}$ vanishes.

As an equation on 1-forms of conformal weight 2 this is in fact described by the first BGGoperator of $\Lambda^{2} \mathcal{T}$ : By (15), the splitting operator

$$
L_{0}^{\Lambda^{2} \mathcal{T}}: \mathfrak{X}(M)=\mathcal{E}_{a}[2] \rightarrow \Lambda^{2} \mathcal{T}=\tilde{\mathcal{A}} M
$$

is given by

$$
\sigma_{a} \in \mathcal{E}_{a}[2] \mapsto\left(\begin{array}{c}
-\frac{1}{10} D^{p} D_{p} \sigma_{a}+\frac{1}{10} D^{p} D_{a} \sigma_{p}-\frac{1}{5} J \sigma_{a} \\
+\frac{2}{5} P_{a}^{p} \sigma_{p}+\frac{1}{25} D_{a} D^{p} \sigma_{p} \\
D_{[c} \sigma_{a]} \mid-\frac{1}{5} D^{p} \sigma_{p} \\
\sigma_{a}
\end{array}\right) .
$$

Now the first BGG-operator $\Theta_{0}^{\Lambda^{2} \mathcal{T}}$ of $\Lambda^{2} \mathcal{T}$ defined by the composition

$$
\Theta_{0}^{\Lambda^{2} \mathcal{T}}: \mathfrak{X}(M)=\mathcal{E}_{a}[2] \rightarrow \mathcal{E}_{(a b)_{0}}[2], \quad \Theta_{0}^{\Lambda^{2} \mathcal{T}}=\Pi_{1} \circ \nabla^{\Lambda^{2} \mathcal{T}} \circ L_{0}^{\Lambda^{2} \mathcal{T}}
$$

is seen by direct calculation employing (14) to be

$$
\xi^{a} \mapsto D_{(c} \xi_{a)_{0}}
$$

for $\xi \in \mathfrak{X}(M)$; i.e., $\Theta_{0}^{\Lambda^{2} \mathcal{T}}$ is the conformally invariant operator governing conformal Killing fields.

We now proceed to prove a technical lemma to be used in the proof of Theorem 4 below. It is a general fact for parabolic geometries satisfying a certain homological condition that parallel sections of the adjoint tractor bundle insert trivially into curvature [9], but it is easy to see this directly for conformal Killing fields, where this has first been observed in [23]. We only sketch a simple proof for conformal structures of dimension $\geq 4$, which is all we need: 
Lemma 7. Let $s \in \Gamma(\tilde{\mathcal{A}} M)$ such that $\nabla^{\tilde{\mathcal{A}}} s=0$. Then $\tilde{K}(\Pi(s), \cdot)=0$.

Proof. Since $\nabla^{\tilde{A}} s=0$ one has $R_{c_{1} c_{2}} s=\nabla_{\left[c_{1}\right.}^{\tilde{A}} \nabla_{\left.c_{2}\right]}^{\tilde{\mathcal{A}}} s=0$, with $R \in \Omega^{2}(M, \mathfrak{g l}(\tilde{\mathcal{A}} M))$ the curvature of $\nabla^{\tilde{\mathcal{A}}}$. But since $\nabla^{\tilde{\mathcal{A}}}$ is the induced tractor connection of the Cartan connection form $\tilde{\omega}$, one has that $R s$ is the algebraic action of $\tilde{K} \in \Omega^{2}(M, \tilde{\mathcal{A}} M)=\Omega^{2}(M, \mathfrak{s o}(\mathcal{T}))$ on $s$; thus, $\tilde{K}$ annihilates $s$. Via the projection $\Pi: \Gamma\left(\Lambda^{2} \mathbf{T}\right) \rightarrow \mathcal{E}_{a}[2]=\mathfrak{X}(M), s$ projects to a conformal Killing field $\sigma^{a} \in \mathfrak{X}(M)$, and from the explicit formula (12) one obtains that the Weyl curvature $C$ annihilates $\sigma$. Employing the symmetries of $C$, one then immediately has that this is equivalent to trivial insertion of $\sigma$. Then $\tilde{K}(\sigma, \cdot)=0$ follows from $(n-2) A_{a b c}=D^{p} C_{p a b c}$.

\subsection{Infinitesimal automorphisms of parabolic geometries}

In this subsection we will use the description [9] of the symmetry Lie algebra of a parabolic geometry $(\mathcal{G}, \omega)$ of type $(G, P)$. This will be applied for $(\mathcal{G}, \omega)$ being, as above, the geometry of type $\left(G_{2}, P\right)$ describing the generic rank 2 distribution $\mathcal{D}$ and for the conformal geometry encoded in the Cartan geometry $(\tilde{\mathcal{G}}, \tilde{\omega})$ of type $(\mathrm{SO}(h), \tilde{P})$. For more details see [9] or [28].

Since $\mathcal{G} \rightarrow M$ is a $P$-principal bundle over $M$ and the geometric structure is encoded in the Cartan connection form $\omega \in \Omega^{1}(\mathcal{G}, \mathfrak{g})$, one defines an automorphism of $(\mathcal{G}, \omega)$ as a $P$ equivariant diffeomorphism $\Psi$ of $\mathcal{G}$ preserving $\omega$, i.e., $\Psi^{*} \omega=\omega$. It is well known that the automorphism group of a Cartan geometry is always a Lie group (see for instance [15]). The space of infinitesimal automorphisms is given by the $P$-invariant vector fields on $\mathcal{G}$ preserving $\omega$, i.e.,

$$
\inf (\mathcal{G}, \omega)=\left\{\xi \in \mathfrak{X}(M)^{P}: \mathcal{L}_{\xi} \omega=0\right\} .
$$

The Lie algebra of the automorphism group of $(\mathcal{G}, \omega)$ then consists of those vector fields in $\inf (\mathcal{G}, \omega)$ that are complete.

Now $\omega: T \mathcal{G} \rightarrow \mathfrak{g}$ is a $P$-equivariant trivialization of $T \mathcal{G}$, and thus, for a $\xi \in \inf (\mathcal{G}, \omega)$ the function $f=\omega \circ \xi: \mathcal{G} \rightarrow \mathfrak{g}$ is $P$-equivariant. The function $f$ therefore defines a section of the adjoint tractor bundle $\mathcal{A} M=\mathcal{G} \times_{P} \mathfrak{g}$.

We have an explicit formula for the tractor connection $\nabla^{\mathcal{A}}$ on $\mathcal{A} M$, see (4): Let $s \in \Gamma(\mathcal{A} M)$ be the section corresponding to the $P$-equivariant function $f \in \mathrm{C}^{\infty}(\mathcal{G}, \mathfrak{g})^{P}$. To compute $\nabla_{\eta}^{\mathcal{A}} s$ for $\eta \in \mathfrak{X}(M)$ we take a $P$-invariant lift $\eta^{\prime} \in \mathfrak{X}(\mathcal{G})$ of $\eta$. Then $\nabla_{\eta}^{\mathcal{A}} s$ corresponds to the $P$-equivariant map

$$
u \mapsto \eta_{u}^{\prime} \cdot \omega(\xi)+\left[\omega_{u}\left(\eta^{\prime}\right), \omega_{u}(\xi)\right] .
$$

For the next lemma, first note that the natural projection

$$
\Pi: \mathcal{A} M=\mathcal{G} \times_{P} \mathfrak{g} \rightarrow \mathcal{G} \times_{P} \mathfrak{g} / \mathfrak{p}=T M
$$

projects $s \in \Gamma(\mathcal{A} M)$ to a vector field $\Pi(s) \in \mathfrak{X}(M)$, which is in fact just the projection of the $P$-invariant vector field $\xi \in \mathfrak{X}(\mathcal{G})^{P}$. Thus one can insert $\Pi(s) \in \mathfrak{X}(M)$ into the curvature form $K \in \Omega^{2}(M, \mathcal{A} M)$.

Proposition $7([9])$. Let $s \in \Gamma(\mathcal{A} M)$ be the adjoint tractor corresponding to the P-invariant vector field $\xi \in \mathfrak{X}(\mathcal{G})^{P}$. Then

$$
\mathcal{L}_{\xi} \omega=0 \text { iff } \nabla_{\eta}^{\mathcal{A}} s+K(\Pi(s), \eta)=0 \forall \eta \in \mathfrak{X}(M),
$$

i.e., $\xi \in \inf (\mathcal{G}, \omega)$ if and only if the corresponding adjoint tractor $s \in \Gamma(\mathcal{A} M)$ is parallel with respect to the connection

$$
\hat{\nabla}^{\mathcal{A}} s=\nabla^{\mathcal{A}} s+K(\Pi(s), \cdot) .
$$




\subsection{Decomposition of the conformal adjoint tractor bundle.}

Let $\mathcal{A} M:=\mathcal{G} \times_{P} \mathfrak{g}$ be the adjoint tractor bundle of a generic 2-distribution $\mathcal{D}$ and let $\tilde{\mathcal{A}} M:=$ $\tilde{\mathcal{G}} \times \tilde{P} \mathfrak{s o}(h)$ be the adjoint tractor bundle of the associated conformal structure. The tractor connection on $\mathcal{A} M$ will be denoted by $\nabla^{\mathcal{A}}$ and the one on $\tilde{\mathcal{A}} M$ by $\nabla^{\tilde{\mathcal{A}}}$.

Recall from Section 4.2 that as a $G_{2}$-representation, $\mathfrak{s o}(h)=\mathbb{R}^{7} \oplus \mathfrak{g}$, i.e., $\mathfrak{s o}(h)$ decomposes into the direct sum of the standard representation of $G_{2} \subset \mathrm{SO}(h)$ on $\mathbb{R}^{7}$ and the adjoint representation Ad $: G_{2} \rightarrow \mathrm{GL}(\mathfrak{g})$. This decomposition was realized by the exact sequence (26) of Section 4.2 and its splitting (27). On the level of associated bundles it yields a decomposition of the conformal adjoint tractor bundle:

$$
\tilde{\mathcal{A}} M=\tilde{\mathcal{G}} \times \tilde{P} \mathfrak{s o}(h)=\mathcal{G} \times{ }_{P} \mathfrak{s o}(h)=\left(\mathcal{G} \times{ }_{P} \mathbb{R}^{7}\right) \oplus\left(\mathcal{G} \times{ }_{P} \mathfrak{g}\right)=\mathcal{T} \oplus \mathcal{A} M .
$$

I.e., the conformal adjoint tractor bundle $\tilde{\mathcal{A}} M$ is the direct sum of the standard tractor bundle $\mathcal{T}$ and the adjoint tractor bundle $\mathcal{A} M$ of the generic distribution.

Let us check that we can also decompose the tractor connection into

$$
\nabla^{\tilde{\mathcal{A}}}=\nabla^{\mathcal{T}} \oplus \nabla^{\mathcal{A}} \text {. }
$$

Take a vector field $\xi \in \mathfrak{X}(M)$ and its horizontal lift $\xi^{\prime}$ to a vector field on the extended bundle $\mathcal{G}^{\prime}=\mathcal{G} \times{ }_{P} G_{2}$. Then, for $s \in \Gamma(\tilde{\mathcal{A}} M)$, the tractor derivative $\nabla_{\xi} s$ is defined by differentiating the $G_{2}$-equivariant function $f: \mathcal{G} \rightarrow \mathfrak{s o}(h)$ corresponding to $s$ in direction $\xi^{\prime}$. But taking this derivative evidently commutes with the algebraic projections of $f$ to its components $f_{\mathcal{T}}: \mathcal{G} \rightarrow \mathbb{R}^{7}$ and $f_{\mathcal{A} M}: \mathcal{G} \rightarrow \mathfrak{g}$; thus $(38)$ holds.

To prove the decomposition of conformal Killing fields we need the following theorem about the deformed connections

$$
\hat{\nabla}^{\mathcal{A}} s=\nabla^{\mathcal{A}} s+K(\Pi(s), \cdot)
$$

and

$$
\hat{\nabla}^{\tilde{\mathcal{A}}} s=\nabla^{\tilde{\mathcal{A}}} s+\tilde{K}(\Pi(s), \cdot)
$$

whose parallel sections describe infinitesimal automorphisms. Recall that according to Lemma 2 we have $\tilde{K}=K \in \Omega^{2}(M, \mathcal{A} M)$.

Theorem 4. Let $s \in \Gamma(\tilde{\mathcal{A}} M)$ be a section of the conformal adjoint tractor bundle which splits according to decomposition $(37)$ into $s_{1} \in \Gamma(\mathcal{T})$ and $s_{2} \in \Gamma(\mathcal{A} M)$. Then $s$ is parallel with respect to $\hat{\nabla}^{\tilde{\mathcal{A}}}$ if and only if $s_{1}$ is $\nabla^{\mathcal{T}}$-parallel and $s_{2}$ is $\hat{\nabla}^{\mathcal{A}}$-parallel.

Proof. Let $s_{1} \in \Gamma(\mathcal{T})$ and $s_{2} \in \Gamma(\mathcal{A} M)$ be $\nabla^{\mathcal{T}}$ - resp. $\hat{\nabla}^{\mathcal{A}}$-parallel sections. Since the restriction of $\nabla^{\tilde{\mathcal{A}}}$ to $\Gamma(\mathcal{T}) \subset \Gamma(\tilde{\mathcal{A}} M)$ is just $\nabla^{\mathcal{T}}$, the section $s_{1}$ includes as a $\nabla^{\tilde{\mathcal{A}}}$-parallel section into $\Gamma(\tilde{\mathcal{A}} M)$. Lemma 7 shows that that $K\left(\Pi\left(s_{1}\right), \cdot\right)=0$, and thus also $\hat{\nabla}^{\tilde{\mathcal{A}}} s_{1}=0$. For $s_{2}$ we have $\hat{\nabla}^{\tilde{\mathcal{A}}} s_{2}=0$ by Lemma 2 , and thus $s:=s_{1}+s_{2}$ satisfies $\hat{\nabla}^{\tilde{\mathcal{A}}} s=0$.

Conversely, we take $s \in \Gamma(\tilde{\mathcal{A}} M)$ with $\hat{\nabla}^{\tilde{\mathcal{A}}} s=0$ and decompose $s=s_{1} \oplus s_{2} \in \Gamma(\mathcal{T}) \oplus \Gamma(\mathcal{A} M)$ according to (37). Since $K$ has values in $\mathcal{A} M$ we have that $s_{1} \in \Gamma(\mathcal{T})$ is parallel with respect to the standard tractor connection $\nabla^{\mathcal{T}}$ by (38). We still need to show that $s_{2}$ is parallel with respect to $\hat{\nabla}^{\mathcal{A}}$, while so far we know that

$$
\nabla^{\mathcal{A}} s_{2}+K\left(\Pi\left(s_{1}\right), \cdot\right)+K\left(\Pi\left(s_{2}\right), \cdot\right)
$$

vanishes. But since $s_{1}$ is parallel as a section of $\tilde{\mathcal{A}} M$ with respect to the usual adjoint tractor connection $\nabla^{\tilde{\mathcal{A}}}$ according to (38), we can again apply Lemma 7 , which tells us that $s_{1}$ inserts trivially into the curvature $\tilde{K}=K$. Thus also $\hat{\nabla}^{\mathcal{A}} s_{2}=0$. 
Proposition 7 provides an identification of $\inf (\tilde{\mathcal{G}}, \tilde{\omega})$ with parallel sections of $\tilde{\mathcal{A}} M$ with respect to $\hat{\nabla}^{\tilde{\mathcal{A}}}$ and an identification of $\inf (\mathcal{G}, \omega)$ with parallel sections of $\mathcal{A} M$ with respect to the connection $\hat{\nabla}^{\mathcal{A}}$. We can thus translate Theorem 4 into a decomposition of conformal Killing fields:

Theorem B. Let $[g]$ be conformal class of signature $(2,3)$-metrics on $M$ and $\phi \in \mathcal{E}_{[a b]}[3]$ a locally decomposable normal conformal Killing two form that satisfies the genericity condition (34). By Theorem $A$, there is a generic distribution $\mathcal{D}$ with $[g]=[g]_{\mathcal{D}}$.

Then every conformal Killing field decomposes into a symmetry of the distribution $\mathcal{D}$ and an almost Einstein scale:

$$
\mathbf{c K f}([g])=\operatorname{sym}(\mathcal{D}) \oplus \mathbf{a E s}([g]) .
$$

The mapping that associates to a conformal Killing field $\xi \in \mathfrak{X}(M)$ its almost Einstein-scale part with respect to the decomposition (39) is given by

$$
\xi_{a} \mapsto \phi_{p q}(D \xi)^{p q}-\frac{1}{2} \xi^{p} D^{q} \phi_{p q},
$$

where $D$ is the Levi Civita connection of an arbitrary metric $g$ in the conformal class.

The mapping that associates to an almost Einstein scale $\sigma \in \mathcal{E}[1]$ a conformal Killing field is given by

$$
\sigma \mapsto \phi_{a p} D^{p} \sigma-\frac{1}{4} \sigma D^{p} \phi_{p a}
$$

Proof. By Proposition 7 conformal Killing fields of $[g]$ are in 1:1-correspondence with $\hat{\nabla}^{\tilde{\mathcal{A}}_{-}}$ parallel sections of $\tilde{\mathcal{A}} M$. By the theorem above every such section decomposes into a parallel standard tractor in $\Gamma(\mathcal{T})$ and a $\hat{\nabla}^{\mathcal{A}}$-parallel section of $\mathcal{A} M$. By Proposition 1 and again Proposition 7, now for $\mathcal{A} M$, this yields the decomposition (39).

It is now straightforward to make this decomposition explicit in terms of the normal conformal Killing 2-form of Theorem A encoding the generic distribution $\mathcal{D}$.

To map an almost Einstein scale $\sigma \in \mathcal{E}[1]$ to a conformal Killing field we use the splitting operator $L_{0}^{\mathcal{T}}: \mathcal{E}[1] \rightarrow \Gamma(\mathcal{T})$ given in (16), contract this section into the characterizing $\nabla^{\Lambda^{3} \mathcal{T}}$ parallel 3-form $\boldsymbol{\Phi} \in \Gamma\left(\Lambda^{3} \mathcal{T}\right)$ given by (33) via the tractor metric $\mathbf{h}$, and project the resulting section of $\Lambda^{2} \mathcal{T}=\tilde{\mathcal{A}} M$ down to $\mathfrak{X}(M)$. This yields (41).

To project a conformal Killing field $\xi \in \mathfrak{X}(M)$ to its almost Einstein scale-part we proceed similarly: we map it to $\Lambda^{2} \mathcal{T}$ via $(36)$, contract it into $\Phi \in \Gamma\left(\Lambda^{3} \mathcal{T}\right)$ and project the resulting standard tractor to $\mathcal{E}[2]$. This gives (40).

To be precise, one has to use a constant scalar multiple of $\phi \in \mathcal{E}_{[a b]}[3]$ such that the composition of (40) with (41) is the identity.

Remark 7. Mapping (41) actually works more generally: in the presence of an almost Einstein scale it was shown in [25, Corollary 5.2] that one can associate to every conformal Killing 2-form, not only to normal ones, a conformal Killing field.

\section{Acknowledgements}

We cannot underestimate the value of discussions with Andreas Čap on various technical procedures used in this paper. The concept of orbit types of parallel tractors was introduced to the first author by Felipe Leitner, who moreover suggested to check for simplicity of the underlying 2 -form. We thank the referees for their careful reading and various valuable suggestions for improvements.

The first author was supported by the IK I008-N funded by the University of Vienna. The second author was supported by project P 19500-N13 of the "Fonds zur Förderung der wissenschaftlichen Forschung" (FWF). 


\section{References}

[1] Alt J., Fefferman constructions in conformal holonomy, Thesis, Humboldt-Universität zu Berlin, 2008.

[2] Armstrong S., Definite signature conformal holonomy: a complete classification, J. Geom. Phys. 57 (2007), 2024-2048, math.DG/0503388.

[3] Bryant R.L., Metrics with exceptional holonomy, Ann. of Math. (2) 126 (1987), 525-576.

[4] Burns D. Jr., Diederich K., Shnider S., Distinguished curves in pseudoconvex boundaries, Duke Math. J. 44 (1977), 407-431.

[5] Calderbank D.M.J., Diemer T., Differential invariants and curved Bernstein-Gelfand-Gelfand sequences, J. Reine Angew. Math. 537 (2001), 67-103, math.DG/0001158.

[6] Čap A., Parabolic geometries, CR-tractors, and the Fefferman construction, Differential Geom. Appl. 17 (2002), 123-138, 2002.

[7] Čap A., Correspondence spaces and twistor spaces for parabolic geometries, J. Reine Angew. Math. 582 (2005), 143-172, math.DG/0102097.

[8] Čap A., Two constructions with parabolic geometries, Rend. Circ. Mat. Palermo (2) Suppl. (2006), no. 79, 11-37, math.DG/0504389.

[9] Čap A., Infinitesimal automorphisms and deformations of parabolic geometries, J. Eur. Math. Soc. (JEMS) 10 (2008), 415-437, math.DG/0508535.

[10] Čap A., Gover A.R., Tractor bundles for irreducible parabolic geometries, in Global Analysis and Harmonic Analysis (Marseille-Luminy, 1999), Sémin. Congr., Vol. 4, Soc. Math. France, Paris, 2000, 129-154.

[11] Čap A., Gover A.R., Tractor calculi for parabolic geometries, Trans. Amer. Math. Soc. 354 (2002), 15111548.

[12] Čap A., Gover A.R., A holonomy characterisation of Fefferman spaces, ESI Preprint 1875, 2006, math.DG/0611939.

[13] Čap A., Gover A.R., CR-tractors and the Fefferman space, Indiana Univ. Math. J. 57 (2008), 2519-2570, math.DG/0611938.

[14] C̆ap A., Sagerschnig K., On Nurowski's conformal structure associated to a generic rank 2 distributions in dimension 5, J. Geom. Phys., to appear, arXiv:0710.2208.

[15] Čap A., Slovák J., Parabolic geometries I: Background and general theory, Vol. 1, Mathematical Surveys and Monographs, American Mathematical Society, Providence, RI, 2009.

[16] Čap A., Slovák J., Souček V., Bernstein-Gelfand-Gelfand sequences, Ann. of Math. (2) 154 (2001), 97-113, math.DG/0001164.

[17] Čap A., Žádník V., On the geometry of chains, J. Differential Geom. 82 (2009), 1-33, math.DG/0504469.

[18] Cartan É., Les espaces á connexion conforme, Ann. Soc. Pol. Math. (1923), no. 2, 172-202, 1923.

[19] Cartan É., Les systèmes de Pfaff, à cinq variables et les équations aux dérivées partielles du second ordre, Ann. Sci. École Norm. Sup. (3) 27 (1910), 109-192.

[20] Doubrov B., Slovák J., Inclusions between parabolic geometries, arXiv:0807.3360.

[21] Eastwood M.G., Michor P.W., Some remarks on the Plücker relations, In The Proceedings of the 19th Winter School "Geometry and Physics" (Srní, 1999), Rend. Circ. Mat. Palermo (2) Suppl. (2000), no. 63, 85-88, math.AG/9905090.

[22] Fefferman C.L., Monge-Ampère equations, the Bergman kernel, and geometry of pseudoconvex domains, Ann. of Math. (2) 103 (1976), 395-416.

[23] Gover A.R., Laplacian operators and Q-curvature on conformally Einstein manifolds, Math. Ann. 336 (2006), 311-334, math.DG/0506037.

[24] Gover A.R., Almost Einstein and Poincaré-Einstein manifolds in Riemannian signature, arXiv:0803.3510.

[25] Gover A.R., Šilhan J., The conformal Killing equation on forms - prolongations and applications, Differential Geom. Appl. 26 (2008), 244-266, math.DG/0601751.

[26] Graham C.R., On Sparling's characterization of Fefferman metrics, Amer. J. Math. 109 (1987), 853-874.

[27] Hammerl M., Natural prolongations of BGG-operators, Thesis, University of Vienna, submitted.

[28] Hammerl M., Homogeneous Cartan geometries, Arch. Math. (Brno) 43 (2007), suppl., 431-442, math.DG/0703627. 
[29] Hammerl M., Invariant prolongation of BGG-operators in conformal geometry, Arch. Math. (Brno) 44 (2008), 367-384, arXiv:0811.4122.

[30] Hitchin N., Stable forms and special metrics, in Global Differential Geometry: the Mathematical Legacy of Alfred Gray (Bilbao, 2000), Contemp. Math., Vol. 288, Amer. Math. Soc., Providence, RI, 2001, 70-89, math.DG/0107101.

[31] Kostant B., Lie algebra cohomology and the generalized Borel-Weil theorem, Ann. of Math. (2) 74 (1961), $329-387$.

[32] Leistner T., Nurowski P., Conformal structures with $G_{2(2)}$-ambient metrics, arXiv:0904.0186.

[33] Leitner F., Conformal Killing forms with normalisation condition, Rend. Circ. Mat. Palermo (2) Suppl. (2005), no. 75, 279-292.

[34] Leitner F., A remark on conformal $\mathfrak{s u}(p, q)$-holonomy, math.DG/0604393.

[35] Leitner F., A remark on unitary conformal holonomy, in Symmetries and Overdetermined Systems of Partial Differential Equations, IMA Vol. Math. Appl., Vol. 144, Springer, New York, 2008, 445-460.

[36] Nurowski P., Differential equations and conformal structures, J. Geom. Phys. 55 (2005), 19-49, math.DG/0406400.

[37] Nurowski P., Conformal structures with explicit ambient metrics and conformal $G_{2}$ holonomy, in Symmetries and Overdetermined Systems of Partial Differential Equations, IMA Vol. Math. Appl., Vol. 144, Springer, New York, 2008, 515-526, math.DG/0701891.

[38] Sagerschnig K., Split octonions and generic rank two distributions in dimension five, Arch. Math. (Brno) 42 (2006), suppl., 329-339.

[39] Sagerschnig K., Weyl structures for generic rank two distributions in dimension five, Thesis, University of Vienna, 2008.

[40] Šilhan J., Cohomologies of real Lie algebras, available at http://bart.math.muni.cz/ silhan/lie/lac/formR.php.

[41] Šilhan J., A real analog of Kostant's version of the Bott-Borel-Weil theorem, J. Lie Theory 14 (2004), 481-499. 